\title{
Mental health training programmes for non-mental health trained professionals coming into contact with people with mental ill health: a systematic review of effectiveness
}

\author{
Alison Booth ${ }^{1 *}$ (D) Arabella Scantlebury ${ }^{1}$, Adwoa Hughes-Morley ${ }^{1}$, Natasha Mitchell ${ }^{1}$, Kath Wright ${ }^{2}$, William Scott ${ }^{3}$ \\ and Catriona McDaid ${ }^{1}$
}

\begin{abstract}
Background: The police and others in occupations where they come into close contact with people experiencing/ with mental ill health, often have to manage difficult and complex situations. Training is needed to equip them to recognise and assist when someone has a mental health issue or learning/intellectual disability. We undertook a systematic review of the effectiveness of training programmes aimed at increasing knowledge, changing behaviour and/or attitudes of the trainees with regard to mental ill health, mental vulnerability, and learning disabilities.

Methods: Databases searched from 1995 onwards included: ASSIA, Cochrane Central Register of Controlled Clinical Trials (CENTRAL), Criminal Justice Abstracts, Embase, ERIC, MEDLINE, PsycINFO, Social Science Citation Index. Courses, training, or learning packages aimed at helping police officers and others who interact with the public in a similar way to deal with people with mental health problems were included. Primary outcomes were change in practice and change in outcomes for the groups of people the trainees come into contact with. Systematic reviews, randomised controlled trials (RCTs) and non- randomised controlled trials (non-RCTs) were included and quality assessed. In addition non-comparative evaluations of training for police in England were included.

Results: From 8578 search results, 19 studies met the inclusion criteria: one systematic review, 12 RCTs, three prospective non-RCTs, and three non-comparative studies.

The training interventions identified included broad mental health awareness training and packages addressing a variety of specific mental health issues or conditions. Trainees included police officers, teachers and other public sector workers. Some short term positive changes in behaviour were identified for trainees, but for the people the trainees came into contact with there was little or no evidence of benefit.

Conclusions: A variety of training programmes exist for non-mental health professionals who come into contact with people who have mental health issues. There may be some short term change in behaviour for the trainees, but longer term follow up is needed. Research evaluating training for UK police officers is needed in which a number of methodological issues need to be addressed.
\end{abstract}

Trial registration: Protocol registration number: PROSPERO: CRD42015015981.

Keywords: Training, Mental health, Police, Systematic review

\footnotetext{
* Correspondence: alison.Booth@york.ac.uk

${ }^{1}$ York Trials Unit, Department of Health Sciences, Faculty of Science,

University of York, Helslington, York YO10 5DD, UK

Full list of author information is available at the end of the article
} 


\section{Background}

Police officers fulfil numerous roles bringing them into close contact with the general public including people with mental ill health [1]. In the UK the police are signatories of the Mental Health Crisis Care Concordat, a multi-agency initiative aimed at improving outcomes for people experiencing mental health crisis [2]. The Concordat recognises the pivotal role played by the police in identifying and deciding on the most appropriate course of action in situations involving individuals with mental ill health. However, police officers are not experts in mental ill health, and often have to manage complex situations with insufficient training. In the UK, gaps in knowledge have been identified, particularly around police officers' understanding of Section 136 of the Mental Health Act 1983, which gives them the power to remove from public places anyone who appears to be suffering from mental disorder and take them to a place of safety [1,3]. Additionally, police officers were not always aware of their responsibilities, even when guidance was provided. The review identified online learning as the main form of training tool for police officers; however there were variations in who had completed the training [1]. Training is a priority for the police, however, the best approach to train officers to respond to people with mental health problems remains unclear. We undertook this systematic review to identify evidence of effectiveness of training approaches to inform the development of a mental health training package for police officers which is currently being assessed in a cluster RCT comparing the new training package with routine training (ISRCTN registry trial ID ISRCTN11685602).

While our main interest was training for police officers, preliminary searches suggested there may be limited studies available specifically in the police. We therefore widened the scope to include non-mental health professionals who may interact with the public in a similar way to the police. Being able to identify problems, deal with situations or refer for professional assistance is expected of people such as teachers and case workers, despite their non-mental health training. It was anticipated that widening the scope would capture a broader range of training approaches that would be relevant in the police setting. We looked at the international literature but as our aim was to inform development of an intervention in the UK, we included a wider range of study designs from the UK to provide context.

We aimed to:

1. Evaluate the evidence on the effectiveness: of training programmes and/or training resources aimed at increasing knowledge and/or changing behaviour or attitudes of the trainees with regard to mental ill health, mental vulnerability, and learning disabilities; and of satisfaction with training and barriers and facilitators to effective training.
2. Identify methods used for evaluating the impact of training interventions.

\section{Methods}

The review followed an a priori protocol: details including minor amendments are available in PROSPERO record CRD42015015981.

\section{Selection criteria}

Participants/population: specific mental health training programmes targeted at police officers; other police staff who come into contact with the public; members of the criminal justice system; non-mental health trained health professionals working in acute health care, including paramedics; people working in education; any other professions, responsible organisations or mental health charities who interact with the public. We excluded basic training delivered to trainees or newly appointed staff in the police force, but included evaluations of additional training. Studies from Organisation for Economic Co-operation and Development (OECD) countries only were included.

Intervention: Training aimed at increasing knowledge of mental health, mental vulnerability, or learning disabilities of members of the public and/or changing attitudes and/or improving their skills in dealing with people with mental health problems in their role, was included. Any courses, training, learning packages or other training resources delivered by any method, for example face-to-face; self-directed; team based or web-based programmes were included. This includes role modelling, reflection, online or mobile phone apps or written materials. Training could focus on the mental health of children, young people and adults.

We used the UK Mental Health Act 2007 definition of mental ill health to encompass, "any disorder or disability of the mind", [3] whether or not a formal diagnosis had been made. Learning disabilities (also called intellectual disabilities) were included in line with the National Policing Improvement Agency guidance on responding to people with mental ill health or learning disabilities [4].

Comparator(s): no-training, usual practice or comparison between different training approaches, for example class room based training vs on-line training.

Outcomes: we were interested in seven possible outcomes of training interventions [5-8]. These were classified into two primary outcomes: change in practice (evaluation of behaviour); and change in outcomes for the groups of people the trainees come into contact with (evaluation of results). The five secondary outcomes were: satisfaction with training (evaluation of reaction), change in attitude towards the importance of mental health, change in confidence, change in knowledge and change in skills (evaluation of learning). 
Types of study included: systematic reviews (that reported their inclusion/exclusion criteria, searched at least one database, provided study details and/or a quality assessment, and synthesised included studies); and RCTs. We included non-RCTs and observational studies without a control group for the police group only, to identify relevant interventions to inform development of the training package. Published and unpublished audits and evaluations of police training in mental health in England and Wales were included.

Qualitative studies of views and experiences of training, and barriers and facilitators to implementation were included to capture satisfaction with training delivery and implementation.

\section{Searches}

An information specialist searched the following bibliographic databases for English language studies from 1995 onwards: ASSIA, Cochrane Central Register of Controlled Clinical Trials (CENTRAL), Criminal Justice Abstracts, Embase, ERIC, MEDLINE, PsycINFO, Social Science Citation Index. The search strategies are available in Additional file 1. We also scanned the complete list of Campbell Reviews and the Register of Studies produced by the Cochrane Effective Practice and Organisation of Care Group. Restricting the searches to the last 20 years took into account the changing legislation attitudes and awareness of mental health.

We checked the reference lists of included studies and of papers that had cited the included papers. The websites of major UK mental health charities were searched (details in Additional file 1). We also searched PROSPERO and ISRCTN for relevant ongoing or completed but not yet published reviews and trials.

Police training officers in England and Wales were asked for their help in identifying published or unpublished audits or evaluations on the impact of mental health training delivered to police officers and/or staff.

\section{Study selection and data collection}

Titles and abstracts of all studies were screened independently by two researchers. Full papers were assessed for inclusion independently by two researchers and at both stages discrepancies were resolved through discussion.

Data extraction forms were developed in Microsoft Excel 2010 and piloted. Data extracted included details of study design (setting, aims, unit of allocation, inclusion criteria, recruitment method etc.), intervention and comparator (type of training, method of delivery, presentation elements, length of training, where delivered, aims of training), and evaluation methods (timing, outcomes measured). Data extraction was undertaken by one researcher and checked by a second; discrepancies were resolved by discussion with a third researcher.
Interventions were classified as didactic if they were exclusively made up of lectures (e.g. presenter determines content, organisation and pace), interactive if they included active participation by the trainees (e.g. case studies, roleplay, group work), and mixed if both were used [5-8].

\section{Risk of bias (quality) assessment}

The ROBIS tool [9] was used to assess the risk of bias of the systematic review; the Cochrane Risk of Bias tool for RCTs [10]; the Newcastle-Ottawa Quality Assessment Scale for Cohort Studies [11]; and the National Institutes for Health tool for studies without a control group [12]. Assessment was undertaken by one researcher and independently checked by a second, with discrepancies resolved by discussion.

\section{Strategy for data synthesis}

We performed a narrative synthesis as a meta-analysis was not feasible due to the substantial differences in interventions and methodological approaches in the included studies. The small number of studies precluded subgroup analysis of short courses as planned in our protocol. Identified qualitative studies provided insight into the barriers, facilitators and perceived impact of training and are presented in a separate paper.

\section{Results}

The search strategies and allied searching identified 8579 references (after deduplication): these were loaded into EndNote $\times 7$ (Thomson Reuters, CA, USA) bibliographic software. Figure 1 shows the flow of studies through the review process. We identified 19 studies for inclusion, and a further eight qualitative studies which are reported separately. Additional file 2 lists the excluded studies.

One systematic review [13], 12 RCTs [14-25] and three prospective non-RCTs of police training [26-28], were included. In addition we included three non-comparative evaluations of mental health related training interventions specifically for the police in England [29-31]. A summary of the characteristics of the included studies is provided in Table 1 .

The studies included in the systematic review were all conducted in the USA. Eight of the 19 primary studies included took place in the USA, three in Sweden, three in England, two in Australia, and one each in Canada, Scotland and Northern Ireland. Participants included teachers, public health professionals, university resident advisors, community practitioners, public sector staff, and case workers. Law enforcement participants were trainee, probationary, university campus, and front line police officers.

Where details of the training venue were reported, this was generally in a training facility environment at participants' place of work: two of the studies held the training 


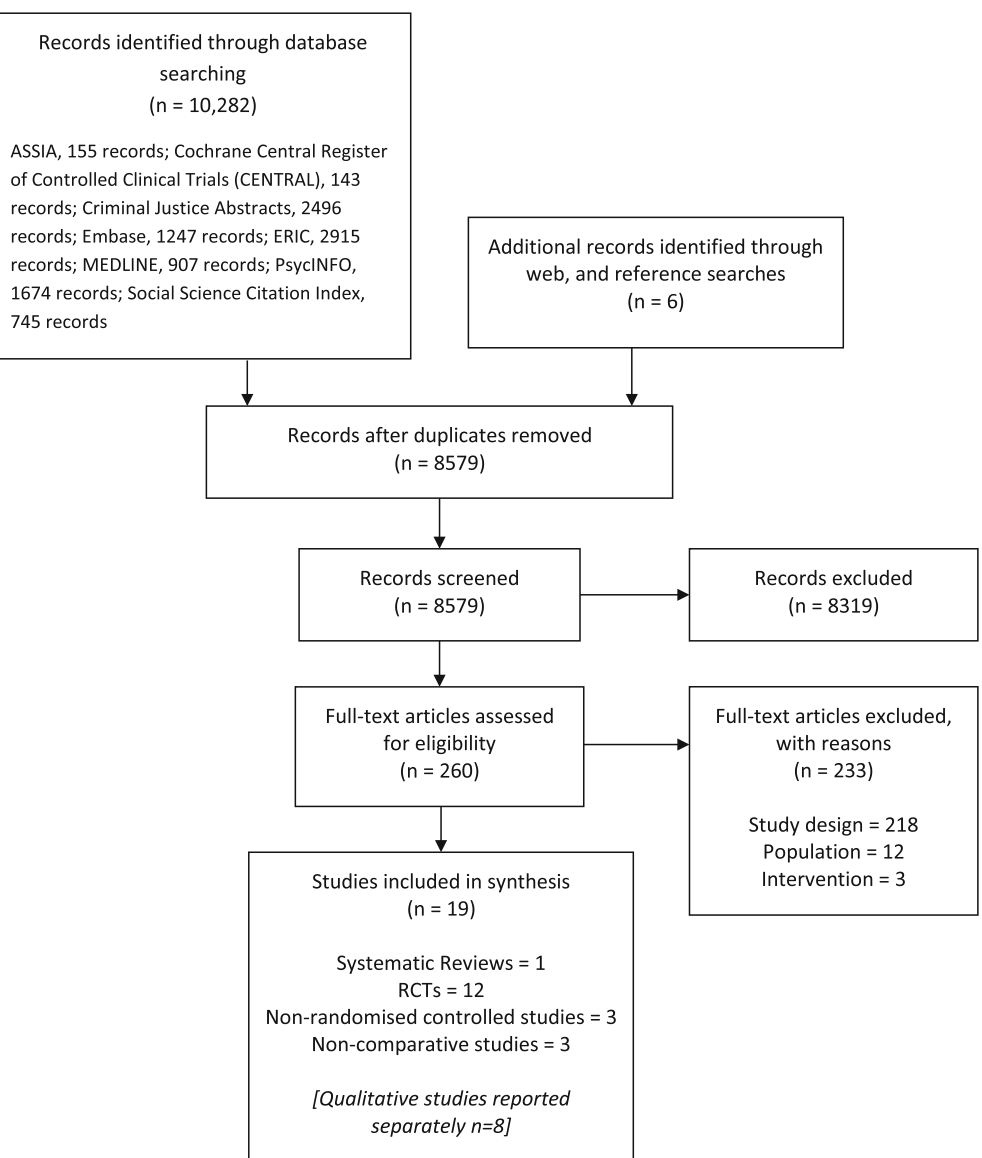

Fig. 1 Flow of studies through review process

in specialist mental health units. Where reported, the number receiving training in a session ranged from 15 to 42 per group. Intervention delivery classed as short (over 1 day or less) ranged from $15 \mathrm{~min}$ to $4 \mathrm{~h}$; and longer delivery times ranged from $12 \mathrm{~h}$ over 2 days to $47 \mathrm{~h}$ with a 1.5 to $2 \mathrm{~h}$ annual booster.

\section{Evaluation of outcomes}

A summary of the measures used for each of the seven outcome categories for this review is provided in Table 2. The main method of evaluation in the included studies was participant completed questionnaire. Reporting of details of the instrument(s) used was generally incomplete. Most studies used a combination of questionnaires to evaluate different aspects of interest. Some validated instruments were used, but most were then modified by the exclusion of questions and/or addition of new questions [20-22, 31]. Many questionnaires were developed by the researchers specifically for their study [14-16, 19-21, 23-25, 29, 30]; most undertook some form of check for internal consistency [14, 19, 20, 22, 24-26].

Three of the RCTs used vignettes combined with a questionnaire to evaluate changes in practice [16], attitude [23], confidence [16], knowledge [25], and behaviour [16, 25]. Other measures used were basic rating scales, for example a categorical measure of the number of residents with whom university resident advisors interacted [21]. None of the included studies undertook an economic evaluation or reported resource use or costs.

\section{Risk of bias within studies}

A summary risk of bias table is provided in Additional file 3 . Overall the included studies were not well reported, in particular, reporting omissions made it difficult to extract or calculate an intervention effect and 95\% confidence interval which would have allowed us to report results across studies.

The systematic review was at high risk of bias [13]. It was unclear whether all the appropriate studies were included in the review, particularly as the authors reported on several studies not identified by their searches. The included studies were not quality assessed and little detail was provided to allow interpretation of the results.

Overall the risk of bias in the RCTs was judged to be unclear because details were not reported, or high risk due to issues with randomisation, blinding or incomplete 
Table 1 Characteristics of included studies

\begin{tabular}{|c|c|c|c|c|c|c|}
\hline Study & Study design & Training evaluated & Participants & Comparator & $\begin{array}{l}\text { Number of } \\
\text { participants } \\
\text { in study }\end{array}$ & $\begin{array}{l}\text { Number of participants in } \\
\text { analysis }\end{array}$ \\
\hline Country & Type of evaluation & & & & $\begin{array}{l}\text { Number of } \\
\text { clusters }\end{array}$ & \\
\hline \multicolumn{7}{|c|}{ Police officers: Interventions with a broad mental health focus } \\
\hline $\begin{array}{l}\text { Compton } \\
\text { (2008) [13] }\end{array}$ & Systematic review & $\begin{array}{l}\text { Crisis Intervention Team } \\
\text { programs (CIT) }\end{array}$ & \multirow[t]{4}{*}{ Police officers } & \multirow{4}{*}{$\begin{array}{l}\text { No } \\
\text { comparators } \\
\text { specified } \\
\text { however the } \\
\text { broad scope } \\
\text { was "research } \\
\text { on CIT" }\end{array}$} & \multirow{4}{*}{$\begin{array}{l}12 \text { studies } \\
\text { were } \\
\text { included in } \\
\text { the review }\end{array}$} & \multirow{4}{*}{$\begin{array}{l}\text { Unclear. No summary table } \\
\text { of studies provided. The } \\
\text { studies are presented in a } \\
\text { narrative synthesis. Some } \\
\text { studies reported number of } \\
\text { participating police officers } \\
\text { and others number of } \\
\text { incidents ( } n=929 \text { police } \\
\text { officers for the } 6 \text { studies of } \\
\text { officer level outcomes) }\end{array}$} \\
\hline \multirow[t]{3}{*}{ USA } & \multirow[t]{3}{*}{$\begin{array}{l}\text { Provide a systematic } \\
\text { summary and critical } \\
\text { analysis of research on } \\
\text { crisis intervention teams }\end{array}$} & $\begin{array}{l}\text { Evaluations, surveys, or } \\
\text { outcome studies conducted } \\
\text { to examine specific questions } \\
\text { or test hypotheses were } \\
\text { included }\end{array}$ & & & & \\
\hline & & $\begin{array}{l}\text { Reports of descriptive } \\
\text { statistics were excluded }\end{array}$ & & & & \\
\hline & & $\begin{array}{l}\text { Searches included: databases } \\
\text { (MEDLINE, PsycINFO, and } \\
\text { databases of criminal justice, } \\
\text { criminology and sociology } \\
\text { abstracts), grey literature } \\
\text { searches, and reference } \\
\text { checking }\end{array}$ & & & & \\
\hline $\begin{array}{l}\text { Forni et al. } \\
\text { (2009) [29] }\end{array}$ & Non-comparative study & \multirow{2}{*}{$\begin{array}{l}\text { Mental health awareness } \\
\text { training to improve } \\
\text { communication and } \\
\text { understanding; explore } \\
\text { common problems and } \\
\text { solutions; understand how } \\
\text { the Mental Health Unit } \\
\text { functions; how MH staff } \\
\text { handle violence and } \\
\text { aggression; understand } \\
\text { common MH problems }\end{array}$} & \multirow[t]{2}{*}{ Police officers } & \multirow[t]{2}{*}{ Not applicable } & \multirow{2}{*}{$\begin{array}{l}364 \\
\text { received } \\
\text { training }\end{array}$} & \multirow[t]{2}{*}{$\begin{array}{l}280 \text { returned evaluation } \\
\text { forms }\end{array}$} \\
\hline England & $\begin{array}{l}\text { Post training evaluation } \\
\text { form }\end{array}$ & & & & & \\
\hline \multirow{2}{*}{$\begin{array}{l}\text { Hansson \& } \\
\text { Markstrom } \\
(2014)[27]\end{array}$} & Prospective non-RCT & \multirow{3}{*}{$\begin{array}{l}\text { An anti-stigma intervention } \\
\text { aimed at improving know- } \\
\text { ledge behaviour and atti- } \\
\text { tudes towards people with } \\
\text { mental illness }\end{array}$} & \multirow[t]{3}{*}{ Police officers } & \multirow[t]{3}{*}{ No training } & \multirow[t]{3}{*}{120} & \multirow{3}{*}{$\begin{array}{l}105 \text { (46 intervention } 59 \\
\text { control) }\end{array}$} \\
\hline & \multirow{2}{*}{$\begin{array}{l}\text { Pre and post intervention } \\
\text { questionnaire. } 6 \text { month } \\
\text { follow up of intervention } \\
\text { group }\end{array}$} & & & & & \\
\hline Sweden & & & & & & \\
\hline \multirow{2}{*}{$\begin{array}{l}\text { Herrington } \\
\& \text { Pope } \\
\text { (2013) [28] }\end{array}$} & Prospective non-RCT & \multirow{4}{*}{$\begin{array}{l}\text { Mental Health Intervention } \\
\text { Team (MHIT): training } \\
\text { uniformed officers as } \\
\text { specialists to respond to } \\
\text { individuals with an apparent } \\
\text { MH concern }\end{array}$} & \multirow{4}{*}{$\begin{array}{l}\text { Front line } \\
\text { police officers } \\
\text { (constables, } \\
\text { senior } \\
\text { constables, } \\
\text { sergeants) }\end{array}$} & \multirow[t]{4}{*}{ No training } & \multirow[t]{4}{*}{185} & \multirow[t]{4}{*}{ Unclear } \\
\hline & $\begin{array}{l}\text { Training group: pre, } 2 \\
\text { months and } 18 \text { months }\end{array}$ & & & & & \\
\hline \multirow[t]{2}{*}{ Australia } & $\begin{array}{l}\text { post intervention } \\
\text { questionnaires }\end{array}$ & & & & & \\
\hline & $\begin{array}{l}\text { Control group: } \\
\text { questionnaire at } \\
18 \text { months only. Routinely } \\
\text { collected data, semi- } \\
\text { structured interviews, ob- } \\
\text { servational data, focus } \\
\text { groups }\end{array}$ & & & & & \\
\hline $\begin{array}{l}\text { Norris \& } \\
\text { Cooke } \\
\text { (2000) [30] }\end{array}$ & Non-comparative study & \multirow{2}{*}{$\begin{array}{l}\text { Training package developed } \\
\text { by MH professionals. Aims to } \\
\text { provide police officers with } \\
\text { understanding, skills and } \\
\text { awareness, and aid the } \\
\text { management of mental } \\
\text { disorder or mental illness }\end{array}$} & \multirow[t]{2}{*}{ Police officers } & \multirow[t]{2}{*}{ Not applicable } & \multirow[t]{2}{*}{$\begin{array}{l}132 \text { officers } \\
\text { trained }\end{array}$} & \multirow[t]{2}{*}{57 responses (43\%) } \\
\hline England & $\begin{array}{l}\text { Immediate post training } \\
\text { evaluation and } \\
\text { retrospective survey }\end{array}$ & & & & & \\
\hline $\begin{array}{l}\text { Pinfold et } \\
\text { al. (2003) } \\
{[31]}\end{array}$ & Non-comparative study & \multirow{2}{*}{$\begin{array}{l}\text { Mental health awareness } \\
\text { training developed in house. } \\
\text { Aim to reduce negative } \\
\text { stereo-typing and discrimin- } \\
\text { atory actions }\end{array}$} & \multirow[t]{2}{*}{ Police officers } & \multirow[t]{2}{*}{ Not applicable } & \multirow{2}{*}{$\begin{array}{l}232 \text { were } \\
\text { eligible to } \\
\text { take part; } \\
163 \text { were } \\
\text { trained }\end{array}$} & \multirow[t]{2}{*}{119 provided follow-up data } \\
\hline England & $\begin{array}{l}\text { Pre and } 4 \text { weeks post } \\
\text { training surveys }\end{array}$ & & & & & \\
\hline
\end{tabular}


Table 1 Characteristics of included studies (Continued)

\begin{tabular}{|c|c|c|c|c|c|c|}
\hline $\begin{array}{l}\text { Rafacz } \\
(2012) \text { [17] }\end{array}$ & $\mathrm{RCT}$ & $\begin{array}{l}\text { Online anti-stigma video } \\
\text { aimed at changing attitudes, }\end{array}$ & $\begin{array}{l}\text { Campus } \\
\text { police officers }\end{array}$ & $\begin{array}{l}\text { Alternative } \\
\text { training }\end{array}$ & 91 & $91^{d}$ \\
\hline USA & $\begin{array}{l}\text { Multiple pre and post } \\
\text { intervention surveys. }\end{array}$ & $\begin{array}{l}\text { reduce stigma and increase } \\
\text { empathy }\end{array}$ & & & & \\
\hline
\end{tabular}

Police officers: Interventions with a specific mental health focus

\begin{tabular}{|c|c|c|c|c|c|c|}
\hline $\begin{array}{l}\text { Bailey et al. } \\
\text { (2001) [26] }\end{array}$ & Prospective non-RCT & \multirow{2}{*}{$\begin{array}{l}\text { Awareness raising training on } \\
\text { intellectual disabilities in } \\
\text { general }\end{array}$} & \multirow[t]{2}{*}{$\begin{array}{l}\text { Police officer } \\
\text { trainees }\end{array}$} & \multirow[t]{2}{*}{ No training } & \multirow[t]{2}{*}{62} & \multirow[t]{2}{*}{$\begin{array}{l}57 \\
\text { (27 intervention } 30 \text { control) }\end{array}$} \\
\hline $\begin{array}{l}\text { Northern } \\
\text { Ireland }\end{array}$ & $\begin{array}{l}\text { Pre and post intervention } \\
\text { questionnaire }\end{array}$ & & & & & \\
\hline $\begin{array}{l}\text { Teagardin } \\
\text { et al. } \\
\text { (2012) [15] }\end{array}$ & Cluster RCT & $\begin{array}{l}\text { Law Enforcement: Your Piece } \\
\text { to the Autism Puzzle. Aims to } \\
\text { help the recognition and }\end{array}$ & $\begin{array}{l}\text { "in the field" } \\
\text { law } \\
\text { enforcement }\end{array}$ & Waiting list & $\begin{array}{l}82 \\
\text { Unclear }\end{array}$ & $81^{e}$ \\
\hline
\end{tabular}

Other non-mental health trained professionals: Interventions with a broad mental health focus

\begin{tabular}{|c|c|c|c|c|c|c|}
\hline $\begin{array}{l}\text { Dorsey et } \\
\text { al. (2012) } \\
{[25]}\end{array}$ & Cluster RCT & \multirow{2}{*}{$\begin{array}{l}\text { Project Focus: training and } \\
\text { consultation model to } \\
\text { improve knowledge of } \\
\text { evidence based practices and } \\
\text { ability to classify } \mathrm{MH} \\
\text { problems and referral options }\end{array}$} & \multirow[t]{2}{*}{$\begin{array}{l}\text { Child welfare } \\
\text { caseworkers }\end{array}$} & \multirow[t]{2}{*}{ Waiting list } & 67 & \multirow[t]{2}{*}{44} \\
\hline USA & $\begin{array}{l}\text { Questionnaire and a } \\
\text { vignette based knowledge } \\
\text { test pre and post } \\
\text { intervention }\end{array}$ & & & & 4 offices & \\
\hline $\begin{array}{l}\text { Jorm et al. } \\
(2010)[23]\end{array}$ & Cluster RCT & \multirow{2}{*}{$\begin{array}{l}\text { A modified version of the } \\
\text { Youth Mental Health First Aid } \\
\text { course. Aims to improve } \\
\text { knowledge, attitudes, } \\
\text { confidence and behaviour for } \\
\text { dealing with students MH } \\
\text { problems }\end{array}$} & \multirow[t]{2}{*}{ Teachers } & \multirow[t]{2}{*}{ Waiting list } & 327 & \multirow[t]{2}{*}{327} \\
\hline Australia & $\begin{array}{l}\text { Pre and post intervention } \\
\text { and 6-month follow up } \\
\text { questionnaire }\end{array}$ & & & & 14 schools & \\
\hline \multirow{2}{*}{$\begin{array}{l}\text { Lipson et } \\
\text { al. (2014) } \\
{[21]}\end{array}$} & \multirow[t]{2}{*}{ Cluster RCT } & \multirow{3}{*}{$\begin{array}{l}\text { Mental Health First Aid. Aims } \\
\text { to improve attitudes and } \\
\text { increase knowledge and self- } \\
\text { efficacy to manage } \mathrm{MH} \text { issues }\end{array}$} & \multirow{3}{*}{$\begin{array}{l}\text { Resident } \\
\text { advisors }\end{array}$} & \multirow[t]{3}{*}{ Usual practice } & 254 & \multirow[t]{3}{*}{2543} \\
\hline & & & & & $\begin{array}{l}32 \text { colleges } \\
\text { and }\end{array}$ & \\
\hline USA & $\begin{array}{l}\text { Surveys } 2-3 \text { months pre } \\
\text { and post training }\end{array}$ & & & & universities & \\
\hline \multirow{2}{*}{$\begin{array}{l}\text { Svensson \& } \\
\text { Hansson } \\
(2014)[16]\end{array}$} & $\mathrm{RCT}$ & \multirow{3}{*}{$\begin{array}{l}\text { Mental Health First Aid } \\
\text { (translated and modified) To } \\
\text { improve MH literacy and give } \\
\text { skills to provide help to } \\
\text { people with MH problems }\end{array}$} & \multirow{3}{*}{$\begin{array}{l}\text { Public sector } \\
\text { staff }\end{array}$} & \multirow[t]{3}{*}{ Waiting list } & \multirow[t]{3}{*}{406} & \multirow[t]{3}{*}{406} \\
\hline & \multirow{2}{*}{$\begin{array}{l}\text { Questionnaires and } \\
\text { vignette } 1 \text { month pre } \\
\text { intervention; } 6 \text { months } \\
\text { post intervention and } \\
2 \text { year follow-up }\end{array}$} & & & & & \\
\hline Sweden & & & & & & \\
\hline $\begin{array}{l}\text { Thombs et } \\
\text { al. (2015) } \\
\text { [14] }\end{array}$ & Cluster RCT & $\begin{array}{l}\text { Peer Hero Training } \\
\text { Programme. Aims to improve } \\
\text { skills, knowledge \& }\end{array}$ & $\begin{array}{l}\text { Resident } \\
\text { assistants }\end{array}$ & Usual practice & 652 & $566^{c}$ \\
\hline USA & $\begin{array}{l}\text { On-line survey at baseline, } \\
\text { follow-ups at 3, } 7 \text { and } \\
9 \text { months }\end{array}$ & $\begin{array}{l}\text { confidence in managing } \\
\text { responses to } \mathrm{MH} \text { and other } \\
\text { situations }\end{array}$ & & & 8 campuses & \\
\hline
\end{tabular}

Other non-mental health trained professionals: Interventions with a specific mental health focus

\begin{tabular}{|c|c|c|c|c|c|c|}
\hline $\begin{array}{l}\text { Hart \& } \\
\text { More } \\
(2013)[24]\end{array}$ & $\mathrm{RCT}$ & \multirow{2}{*}{$\begin{array}{l}\text { Audio-visual synchronous } \\
\text { podcast of information } \\
\text { related to Autism Spectrum } \\
\text { Disorder (ASD) with the aim } \\
\text { of improving knowledge }\end{array}$} & \multirow[t]{2}{*}{$\begin{array}{l}\text { Trainee } \\
\text { teachers }\end{array}$} & \multirow[t]{2}{*}{$\begin{array}{l}\text { Alternative } \\
\text { training }\end{array}$} & \multirow[t]{2}{*}{79} & \multirow[t]{2}{*}{79} \\
\hline USA & $\begin{array}{l}\text { Pre and post intervention } \\
\text { survey }\end{array}$ & & & & & \\
\hline $\begin{array}{l}\text { Kolko et al. } \\
(2012)[22]\end{array}$ & $\mathrm{RCT}$ & \multirow{2}{*}{$\begin{array}{l}\text { Alternatives for Families: A } \\
\text { Cognitive-Behavioral Therapy } \\
\text { (AF-CBT). Aims to provide } \\
\text { skills to use AF-CBT when } \\
\text { working with families with }\end{array}$} & \multirow[t]{2}{*}{$\begin{array}{l}\text { Community } \\
\text { practitioners }\end{array}$} & \multirow[t]{2}{*}{ Usual practice } & \multirow[t]{2}{*}{182} & \multirow[t]{2}{*}{128} \\
\hline JSA & $\begin{array}{l}\text { Questionnaires completed } \\
\text { at baseline, 6, } 12 \text { and } \\
18 \text { months }\end{array}$ & & & & & \\
\hline
\end{tabular}


Table 1 Characteristics of included studies (Continued)

\begin{tabular}{|c|c|c|c|c|c|c|}
\hline & & $\begin{array}{l}\text { physical forces, aggression or } \\
\text { abuse of children }\end{array}$ & & & & \\
\hline $\begin{array}{l}\text { McVey et } \\
\text { al. (2008) } \\
{[20]}\end{array}$ & Cluster RCT & \multirow{2}{*}{$\begin{array}{l}\text { The student body: promoting } \\
\text { health at any size an online } \\
\text { programme." Aims to help } \\
\text { prevent the onset of } \\
\text { disordered eating through } \\
\text { the promotion of positive } \\
\text { body image }\end{array}$} & \multirow[t]{2}{*}{$\begin{array}{l}\text { Teachers and } \\
\text { public health } \\
\text { professionals }\end{array}$} & \multirow[t]{2}{*}{ Waiting list } & 167 & \multirow[t]{2}{*}{$85^{b}$} \\
\hline Canada & $\begin{array}{l}\text { Pre and } 60 \text { days post } \\
\text { intervention questionnaire }\end{array}$ & & & & $\begin{array}{l}37 \text { schools } \\
\text { and their } \\
\text { public } \\
\text { health } \\
\text { agencies } \\
\text { (number } \\
\text { unclear) }\end{array}$ & \\
\hline $\begin{array}{l}\text { Moor et al. } \\
\text { (2007) [19] }\end{array}$ & RCT & \multirow{2}{*}{$\begin{array}{l}\text { Educational package on } \\
\text { recognition, identification } \\
\text { and attitudes towards } \\
\text { adolescent depression }\end{array}$} & \multirow[t]{2}{*}{ Teachers } & \multirow[t]{2}{*}{ Waiting list } & \multirow[t]{2}{*}{151} & \multirow[t]{2}{*}{151} \\
\hline Scotland & $\begin{array}{l}\text { Pre and post training } \\
\text { questionnaire }\end{array}$ & & & & & \\
\hline $\begin{array}{l}\text { Ostberg \& } \\
\text { Rydell } \\
\text { (2012) [18] }\end{array}$ & $\mathrm{RCT}$ & \multirow{2}{*}{$\begin{array}{l}\text { A modified version of } \\
\text { Barkley's parent training } \\
\text { programme. Aims to provide } \\
\text { tools and strategies to help } \\
\text { children with Attention } \\
\text { Deficit Hyperactivity Disorder } \\
\text { (ADHD) }\end{array}$} & \multirow[t]{2}{*}{$\begin{array}{l}\text { Parents and } \\
\text { teachers }\end{array}$} & \multirow[t]{2}{*}{ Waiting list } & \multirow[t]{2}{*}{147} & \multirow[t]{2}{*}{$129^{a}$} \\
\hline Sweden & $\begin{array}{l}\text { Pre and post intervention } \\
\text { and } 3 \text {-month follow up } \\
\text { questionnaire }\end{array}$ & & & & & \\
\hline
\end{tabular}

${ }^{\mathrm{a}}$ For most but not all analyses. ${ }^{\mathrm{b}}$ Calculated from \% of non-completers. ${ }^{\mathrm{C}}$ The authors state results for 566 are reported, but Fig. 1 shows total of 565 at baseline measures. ${ }^{\mathrm{d}} 91$ completed initial part; 52 completed follow up part; data were imputed for missing responses. ${ }^{\text {e }}$ One control participant withdrew following second pre-test

outcome data [14-25]. The risk of bias in the non-RCTs was generally unclear, mainly because of inadequate reporting of the methods [26-28]. The quality rating for one of the non-comparative studies was good [31] and the other two had reporting omissions, but were judged as fair [29, 30].

\section{Training interventions}

The interventions evaluated in the review [13], four of the RCTs [16, 18, 21, 23] and one non-RCT [28] were based on established training programmes used in other settings. The remaining studies evaluated training which the authors had devised or were involved in developing. Descriptions of the training interventions and their delivery were generally insufficient for reproduction. However, details of the established programmes are available in related publications and four others online $[14,15,20,22]$. Where details of those delivering the training were reported they generally included trainers with experience of working in mental health, such as social workers. The studies of police officers nearly all included mental health workers and police trainers, but only one also included service users as trainers [31]. Characteristics of the training interventions are given in Table 3.

\section{Training for police officers: Interventions with a broad mental health focus}

The systematic review focussed on CIT programmes originating in Memphis, USA. CIT is a police based response undertaken in collaboration with other services such as mental health professionals and ambulance services. These programmes provide selected officers with specialist training in dealing with mental health related calls. CIT/MHIT trained officers then provide a specialised front-line response to calls with the aim of directing those with mental health problems to treatment services rather than the judicial system.

A review of CIT programs (Compton, 2008) reported that CIT may be an effective component in connecting individuals with mental illnesses who come to the attention of police officers with appropriate psychiatric services. The limited and poor quality research identified indicates that the training component of the CIT model may have a positive effect on officers' attitudes, beliefs, and knowledge relevant to interactions with people with mental health problems. CIT-trained officers have reported feeling better prepared in handling calls involving individuals with mental illnesses [13]. On a systems level, the review found that CIT, in comparison to other pre- and post-diversion programs, may be associated with a lower arrest rate and lower associated criminal justice costs [13]. However, the review failed to find support for the roll out of CIT.

In a non-RCT, Herrington et al. (2014) evaluated the MHIT programme in Australia [28] which is based on the CIT model. There was little change in practice or perceived quality of relationships between the police and other stakeholders [28]. There were no significant differences between the MHIT trained and non-MHIT trained officers in terms of skills, except once trained, MHIT 
Table 2 Outcome measures used

\begin{tabular}{|c|c|c|c|c|c|c|c|}
\hline & Primary outcomes & & Secondary outcon & & & & \\
\hline Study & Change in practice & $\begin{array}{l}\text { Change in } \\
\text { outcomes for } \\
\text { the groups of } \\
\text { people the } \\
\text { trainees come } \\
\text { into contact } \\
\text { with }\end{array}$ & $\begin{array}{l}\text { Satisfaction with } \\
\text { training: }\end{array}$ & $\begin{array}{l}\text { Change in } \\
\text { attitude towards } \\
\text { the importance of } \\
\text { mental health }\end{array}$ & $\begin{array}{l}\text { Change in } \\
\text { confidence }\end{array}$ & $\begin{array}{l}\text { Change in } \\
\text { knowledge: }\end{array}$ & $\begin{array}{l}\text { Change in skills } \\
\text { - change in } \\
\text { behaviour/ } \\
\text { application of } \\
\text { skills: }\end{array}$ \\
\hline \multicolumn{8}{|c|}{ Police officers: Interventions with a broad mental health focus } \\
\hline $\begin{array}{l}\text { Forni et al. } \\
\text { (2009) [29] }\end{array}$ & Not measured & Not measured & $\begin{array}{l}\text { Feedback form } \\
\text { evaluated quality } \\
\text { of presentation } \\
\text { and content }\end{array}$ & $\begin{array}{l}\text { Form evaluated } \\
\text { quality of } \\
\text { presentation and } \\
\text { content }\end{array}$ & Not measured & $\begin{array}{l}\text { Form evaluated } \\
\text { quality of } \\
\text { presentation and } \\
\text { content }\end{array}$ & $\begin{array}{l}\text { Form evaluated } \\
\text { quality of } \\
\text { presentation } \\
\text { and content }\end{array}$ \\
\hline \multirow[t]{2}{*}{$\begin{array}{l}\text { Hansson \& } \\
\text { Markstrom } \\
(2014)[27]\end{array}$} & Not measured & Not measured & Not measured & $\begin{array}{l}\text { Attitudes: } \\
\text { Community } \\
\text { Attitudes towards } \\
\text { Mental Illness. } \\
\text { Validated. } 20 \\
\text { items. Score: 6- } \\
\text { point Likert scale. }\end{array}$ & Not measured & $\begin{array}{l}\text { Mental Health } \\
\text { Knowledge Scale. } \\
\text { Validated } 12 \\
\text { items, scored on } \\
1-5 \text { scale }\end{array}$ & Not measured \\
\hline & & & & $\begin{array}{l}\text { Behaviour: } \\
\text { Reported and } \\
\text { Intentional } \\
\text { Behaviour Scale: } \\
\text { Validated. } 8 \text { items: } \\
4 \text { scored Yes/No } \\
\text { and } 4 \text { on 1-5 } \\
\text { scale }\end{array}$ & & & \\
\hline $\begin{array}{l}\text { Herrington } \\
\text { \& Pope } \\
\text { (2013) [28] }\end{array}$ & $\begin{array}{l}\text { Surveys. Number } \\
\text { of items and } \\
\text { validation not } \\
\text { reported }\end{array}$ & $\begin{array}{l}\text { Surveys. } \\
\text { Number of } \\
\text { items and } \\
\text { validation not } \\
\text { reported }\end{array}$ & $\begin{array}{l}\text { Surveys. Number } \\
\text { of items and } \\
\text { validation not } \\
\text { reported }\end{array}$ & Not measured & $\begin{array}{l}\text { No comparative } \\
\text { data reported }\end{array}$ & Not measured & $\begin{array}{l}\text { Surveys. } \\
\text { Number of } \\
\text { items and } \\
\text { validation not } \\
\text { reported }\end{array}$ \\
\hline $\begin{array}{l}\text { Norris \& } \\
\text { Cooke } \\
(2000)[30]\end{array}$ & $\begin{array}{l}\text { Questionnaire } \\
\text { developed by } \\
\text { study team }\end{array}$ & Not measured & Not measured & Not measured & Not measured & Not measured & Not measured \\
\hline $\begin{array}{l}\text { Pinfold et } \\
\text { al. (2003) } \\
{[31]}\end{array}$ & $\begin{array}{l}\text { Modified } \\
\text { Community } \\
\text { Attitudes towards } \\
\text { Mental Illness } \\
\text { (CAMI) and World } \\
\text { Psychiatric } \\
\text { Association (WPA } \\
\text { 2000) } \\
\text { questionnaires. } \\
\text { Scored on } 5 \text { point } \\
\text { likert scale }\end{array}$ & Not measured & $\begin{array}{l}\text { One page } \\
\text { feedback form }\end{array}$ & $\begin{array}{l}\text { Modified CAMI } \\
\text { and WPA } 2000 \\
\text { questionnaires. } \\
\text { Scored on } 5 \text { point } \\
\text { likert scale }\end{array}$ & $\begin{array}{l}\text { Modified CAMI } \\
\text { and WPA } 2000 \\
\text { questionnaires. } \\
\text { Scored on } 5 \text { point } \\
\text { likert scale }\end{array}$ & $\begin{array}{l}\text { Modified CAMI } \\
\text { and WPA } 2000 \\
\text { questionnaires. } \\
\text { Scored on } 5 \text { point } \\
\text { likert scale }\end{array}$ & $\begin{array}{l}\text { Modified CAMI } \\
\text { and WPA } 2000 \\
\text { questionnaires. } \\
\text { Scored on } 5 \\
\text { point likert scale }\end{array}$ \\
\hline $\begin{array}{l}\text { Rafacz } \\
(2012)[17]\end{array}$ & Not measured & Not measured & Not measured & $\begin{array}{l}\text { Modified } \\
\text { Attribution } \\
\text { Questionnaire. } 31 \\
\text { questions, } \\
\text { response on a 5- } \\
\text { point Likert scale }\end{array}$ & Not measured & Not measured & Not measured \\
\hline \multicolumn{8}{|c|}{ Police officers: Interventions with a specific mental health focus } \\
\hline $\begin{array}{l}\text { Bailey et al. } \\
\text { (2001) [26] }\end{array}$ & Not measured & Not measured & Not measured & $\begin{array}{l}\text { Attitudes } \\
\text { towards Mental } \\
\text { Retardation and } \\
\text { Eugenics } \\
\text { questionnaire. } \\
\text { Validated. } 32 \\
\text { items measured } \\
\text { on a Likert scale. } \\
\text { Score range 32- } \\
192\end{array}$ & Not measured & Not measured & Not measured \\
\hline
\end{tabular}


Table 2 Outcome measures used (Continued)

\begin{tabular}{|c|c|c|c|c|c|c|c|}
\hline $\begin{array}{l}\text { Teagardin } \\
\text { et al. } \\
\text { (2012) [15] }\end{array}$ & Not measured & Not measured & Not measured & Not measured & $\begin{array}{l}2 \text { of a } 12 \text { item } \\
\text { questionnaire } \\
\text { developed by } \\
\text { study team: } \\
\text { scored on Likert } \\
\text { scale }\end{array}$ & $\begin{array}{l}10 \text { of a } 12 \text { item } \\
\text { questionnaire } \\
\text { developed by } \\
\text { study team: } \\
\text { scored on Likert } \\
\text { scale }\end{array}$ & Not measured \\
\hline
\end{tabular}

Other non-mental health trained professionals: Interventions with a broad mental health focus

\begin{tabular}{|c|c|c|c|c|c|c|c|}
\hline $\begin{array}{l}\text { Dorsey et } \\
\text { al. (2012) } \\
{[25]}\end{array}$ & $\begin{array}{l}54 \text { item } \\
\text { questionnaire } \\
\text { developed by } \\
\text { study team }\end{array}$ & Not measured & Not measured & Not measured & Not measured & $\begin{array}{l}\text { Vignette-based } \\
\text { knowledge test } \\
\text { developed by } \\
\text { study team }\end{array}$ & $\begin{array}{l}\text { Vignette-based } \\
\text { knowledge test } \\
\text { developed by } \\
\text { study team }\end{array}$ \\
\hline $\begin{array}{l}\text { Jorm et al. } \\
\text { (2010) [23] }\end{array}$ & Not measured & $\begin{array}{l}\text { Strengths \& } \\
\text { Difficulties } \\
\text { Questionnaire: } \\
\text { validated } \\
\text { measure of } 25 \\
\text { items: score } \\
\text { range } 0-3\end{array}$ & Not measured & $\begin{array}{l}\text { Vignette-based } \\
\text { knowledge test } \\
\text { developed by } \\
\text { study team. Non- } \\
\text { validated }\end{array}$ & $\begin{array}{l}\text { Two item } \\
\text { questionnaire } \\
\text { developed by the } \\
\text { study team. Non- } \\
\text { validated. Score } \\
\text { range } 0-5\end{array}$ & $\begin{array}{l}21 \text { item } \\
\text { questionnaire } \\
\text { developed by the } \\
\text { study team. Non- } \\
\text { validated. Score } \\
\text { range } 0-3\end{array}$ & $\begin{array}{l}4 \text { item } \\
\text { questionnaire } \\
\text { developed by } \\
\text { the study team. } \\
\text { Non-validated. } \\
\text { Score range 0-4 }\end{array}$ \\
\hline $\begin{array}{l}\text { Lipson et } \\
\text { al. (2014) } \\
{[21]}\end{array}$ & $\begin{array}{l}\text { Study team } \\
\text { produced } \\
\text { categorical } \\
\text { measure of the } \\
\text { number of } \\
\text { residents with } \\
\text { whom Resident } \\
\text { Advisors discussed } \\
\text { mental health } \\
\text { issues }(0,1,2-3 \text {, or } \\
4+) ;\end{array}$ & $\begin{array}{l}\text { The K6 scale } \\
\text { for } \\
\text { psychological } \\
\text { distress. } \\
\text { Validated } \\
\text { scale. Score } \\
\text { range: } 0 \text { to } 24\end{array}$ & Not measured & $\begin{array}{l}3 \text { item measure } \\
\text { of personal } \\
\text { stigma adapted } \\
\text { from the existing } \\
\text { Discrimination- } \\
\text { Devaluation Scale. } \\
\text { Score range: } 0-5\end{array}$ & $\begin{array}{l}\text { Measure used was } \\
\text { not clearly } \\
\text { reported }\end{array}$ & $\begin{array}{l}\text { Non-validated } \\
\text { question } \\
\text { developed by the } \\
\text { researchers. } 5 \\
\text { response } \\
\text { categories }\end{array}$ & $\begin{array}{l}\text { Non-validated } 6 \\
\text { items related to } \\
\text { self-perceived } \\
\text { gatekeeper skills. }\end{array}$ \\
\hline $\begin{array}{l}\text { Svensson \& } \\
\text { Hansson } \\
(2014)[16]\end{array}$ & $\begin{array}{l}\text { Pre-existing } \\
\text { Questionnaires } \\
\text { with vignettes. } \\
\text { Vignettes scored } \\
\text { max. } 5 \text { or } 6\end{array}$ & Not measured & Not measured & $\begin{array}{l}\text { Pre-existing Social } \\
\text { distance scale. } 6 \\
\text { items, scored on } \\
\text { a } 1-3 \text { scale. }\end{array}$ & $\begin{array}{l}\text { Pre-existing } \\
\text { Questionnaires } \\
\text { with vignettes. } \\
\text { Vignettes scored } \\
\text { max. } 5 \text { or } 6\end{array}$ & $\begin{array}{l}\text { Non-validated } \\
\text { Questionnaire } \\
\text { developed by } \\
\text { study team. } 16 \\
\text { items, response } \\
\text { scale } 0-2 . \text { Score } \\
\text { range } 0-16\end{array}$ & $\begin{array}{l}\text { Pre-existing } \\
\text { Questionnaires } \\
\text { with vignettes. } \\
\text { Vignettes scored } \\
\text { max. } 5 \text { or } 6\end{array}$ \\
\hline $\begin{array}{l}\text { Thombs et } \\
\text { al. (2015) } \\
\text { [14] }\end{array}$ & $\begin{array}{l}12 \text { item survey } \\
\text { developed by } \\
\text { study team } \\
\text { measured the } \\
\text { number of first aid } \\
\text { efforts made over } \\
\text { the previous } \\
30 \text { days }\end{array}$ & Not measured & Not measured & Not measured & $\begin{array}{l}\text { Referral efficacy, } \\
\text { scored on a 5- } \\
\text { point Likert scale }\end{array}$ & Not measured & Not measured \\
\hline
\end{tabular}

Other non-mental health trained professionals: Interventions with a specific mental health focus

\begin{tabular}{|c|c|c|c|c|c|c|c|}
\hline $\begin{array}{l}\text { Hart \& } \\
\text { More } \\
(2013)[24]\end{array}$ & Not measured & Not measured & Not measured & Not measured & Not measured & $\begin{array}{l}\text { Non-validated } \\
\text { questionnaire, } \\
\text { with ten open- } \\
\text { ended questions: } \\
\text { score range 0-3 }\end{array}$ & Not measured \\
\hline $\begin{array}{l}\text { Kolko et al. } \\
\text { (2012) [22] }\end{array}$ & $\begin{array}{l}35 \text { item AF-CBT } \\
\text { implementation } \\
\text { measure (AIM). } \\
\text { Scores rated on a } \\
\text { 5-point Likert-type } \\
\text { scale }\end{array}$ & Not measured & $\begin{array}{l}\text { 13-item training } \\
\text { evaluation } \\
\text { developed by The } \\
\text { National Child } \\
\text { Trauma Stress } \\
\text { Network. } 11 \text { Items }\end{array}$ & Not measured & Not measured & $\begin{array}{l}\text { 25-item CBT } \\
\text { Knowledge } \\
\text { Questionnaire. } \\
\text { Multiple-choice: } 4 \\
\text { or } 5 \text { response } \\
\text { choices. }\end{array}$ & $\begin{array}{l}35 \text { item AF-CBT } \\
\text { implementation } \\
\text { measure (AIM). } \\
\text { Scores rated on } \\
\text { a 5-point Likert- } \\
\text { type scale }\end{array}$ \\
\hline
\end{tabular}


Table 2 Outcome measures used (Continued)

\begin{tabular}{|c|c|c|c|c|c|c|c|}
\hline $\begin{array}{l}\text { McVey et } \\
\text { al. (2008) } \\
{[20]}\end{array}$ & Not measured & Not measured & $\begin{array}{l}\text { A } 24 \text { item non- } \\
\text { validated question- } \\
\text { naire developed } \\
\text { by study team. a } \\
\text { 4-point, forced- } \\
\text { choice scale }\end{array}$ & $\begin{array}{l}\text { 6-item } \\
\text { questionnaire } \\
\text { developed by } \\
\text { study team, rated } \\
\text { on a 4-point } \\
\text { Likert scale }\end{array}$ & $\begin{array}{l}\text { 6-item subscale } \\
\text { from existing } \\
\text { validated "Free to } \\
\text { Be Me" survey, } \\
\text { adapted for } \\
\text { teachers. Score: 4- } \\
\text { point Likert scale }\end{array}$ & $\begin{array}{l}\text { Validated survey } \\
\text { "Free to Be Me" } \\
\text { (modified); in- } \\
\text { house survey; } \\
\text { items, from Per- } \\
\text { ceived Media In- } \\
\text { fluences Sub- } \\
\text { Scale. Scored on a } \\
\text { 4/5-point Likert } \\
\text { scale }\end{array}$ & $\begin{array}{l}\text { 6-item subscale } \\
\text { from existing } \\
\text { validated "Free } \\
\text { to Be Me" } \\
\text { survey, adapted } \\
\text { for teachers. } \\
\text { Score: 4-point } \\
\text { Likert scale }\end{array}$ \\
\hline $\begin{array}{l}\text { Moor et al. } \\
\text { (2007) [19] }\end{array}$ & $\begin{array}{l}\text { Percentage of } \\
\text { pupils reported as } \\
\text { depressed by } \\
\text { teachers, using lists } \\
\text { of class cohort }\end{array}$ & Not measured & Not measured & Not measured & $\begin{array}{l}\text { Attitudes } \\
\text { Questionnaire } \\
\text { designed by the } \\
\text { research team. } \\
\text { This was piloted } \\
\text { however unclear } \\
\text { to what extent it } \\
\text { was validated. } \\
30 \text { mins to } \\
\text { complete }\end{array}$ & Not measured & $\begin{array}{l}\text { Lists of class } \\
\text { cohort }\end{array}$ \\
\hline $\begin{array}{l}\text { Ostberg \& } \\
\text { Rydell } \\
\text { (2012) [18] }\end{array}$ & Not measured & $\begin{array}{l}\text { ADHD Rating } \\
\text { Scale 0-4; } \\
\text { DSM-IV scale } \\
\text { 0-4; Strengths } \\
\text { and Difficulties } \\
\text { Questionnaire } \\
\text { validated, 20- } \\
\text { items, scale 1-5 }\end{array}$ & $\begin{array}{l}\text { Described by } \\
\text { authors as } \\
\text { measured, but } \\
\text { how was not } \\
\text { reported }\end{array}$ & Not measured & Not measured & Not measured & Not measured \\
\hline
\end{tabular}

N.B. Number of items and scoring are included in the table where they were available. None of the studies included an economic evaluation or reported resource use or costs

officers reported spending less time at Mental Health Act events (trained mean 54.5mins; control 99.5 mins).

Two non-comparative studies reported on local collaborations between police forces and professionals in mental health units delivering 1 day mental health awareness training to front line police officers [29, 30]. Forni's (2009) post training survey found a high degree of satisfaction with the training and officers said it was relevant to their daily work [29]. They also self-reported better understanding of mental health services with some myths being dispelled and terms such as 'psychotic' and 'delusions' becoming clearer. The mental health professionals who delivered the training also reported a better understanding of the role of the police and the pressures and constraints they operate under. Norris and Cooke (2000) reported a retrospective survey which aimed to establish how useful the training had been in practice [30]. Of the 55 respondents, 53 (96\%) had dealt with mentally ill people, 34 (61\%) had used their training and $37(67 \%)$ felt the training had increased their ability to deal with people with mental illness. Having regular updates was felt necessary by 15 (27\%) respondents.

Hansson and Markstrom's (2014) non-RCT assessed an anti-stigma course as an addition to the regular police officer training psychiatry course [27]. Rafacz (2012) compared two ways of presenting an on-line anti-stigma programme to campus police officers in a non-RCT: personal experience versus information giving [17]. A non- comparative evaluation of an educational intervention to reduce psychiatric stigma and discrimination in the police force in England was undertaken by Pinfold et al. (2014) [31]. All three studies took a team based approach and used mixed teaching methods. Hansson and Markstrom (2014) and Pinfold (2014) delivered face-to-face training and included people with experience of mental health problems as trainers. Rafacz (2012) used two video presentations: one where the presenter disclosed his mental illness and the other with no disclosure.

Hansson and Markstrom (2014) found improved attitudes, mental health literacy and knowledge, and an increased willingness to interact with people with mental illness post intervention. Improvements were also seen at a 6 month follow-up; however this was based on data from the intervention group only [27]. In comparing antistigma videos of personal experience with information giving, Rafacz (2012) found neither was effective in changing attitudes [17]. The analysis suggested that attitudes of the campus police officers were generally non-stigmatising.

The non-comparative evaluation by Pinfold et al. (2003) included a pre and immediate post training satisfaction survey and a 4-week post training survey (data from 109 officers). The training aimed to reduce psychiatric stigma and discrimination [31]. A positive impact on police work post training was identified by 32 (59\%) police officers, mostly through a clearer understanding leading to better communications; while $22(41 \%)$ felt the training had 
Table 3 Characteristics of training interventions

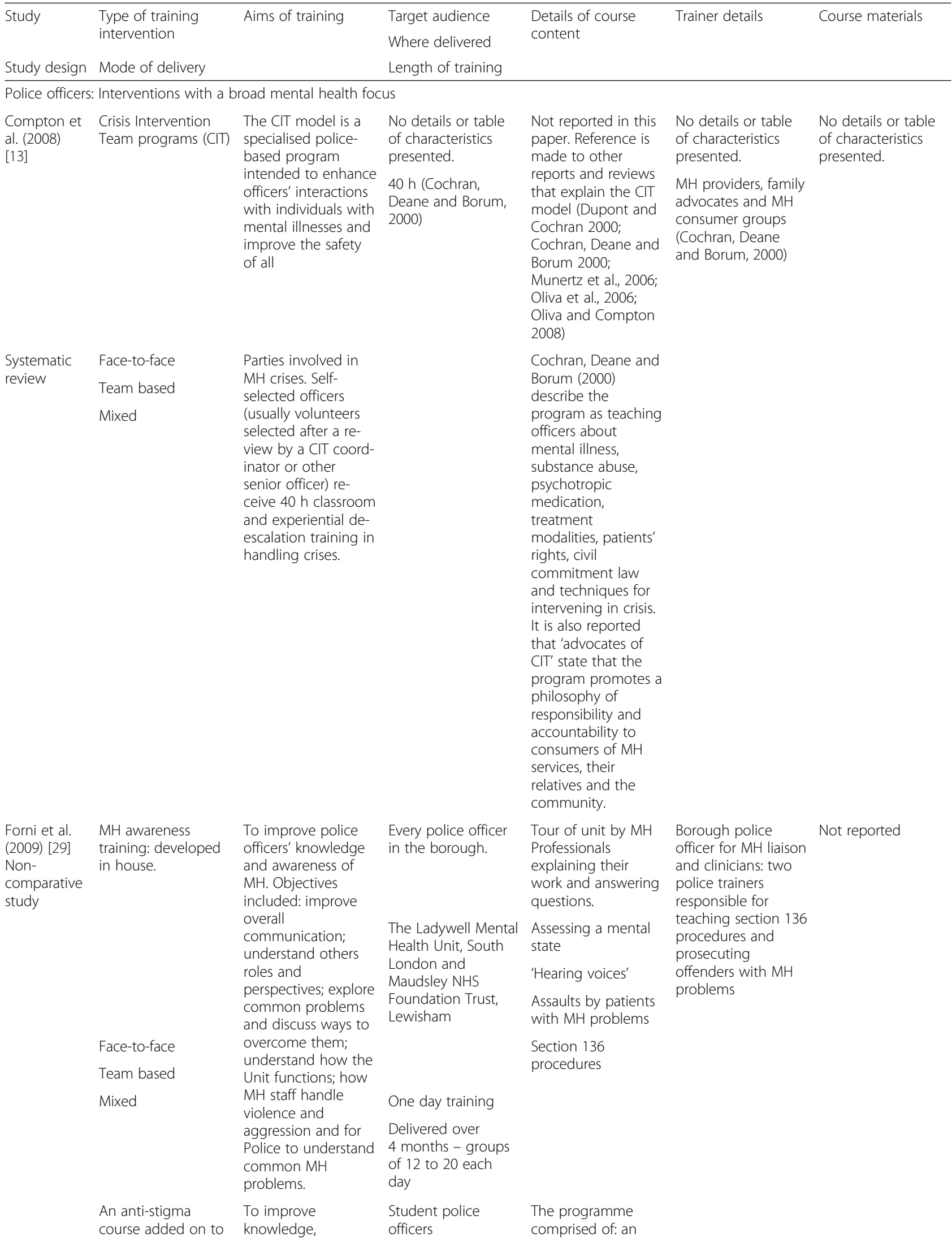


Table 3 Characteristics of training interventions (Continued)

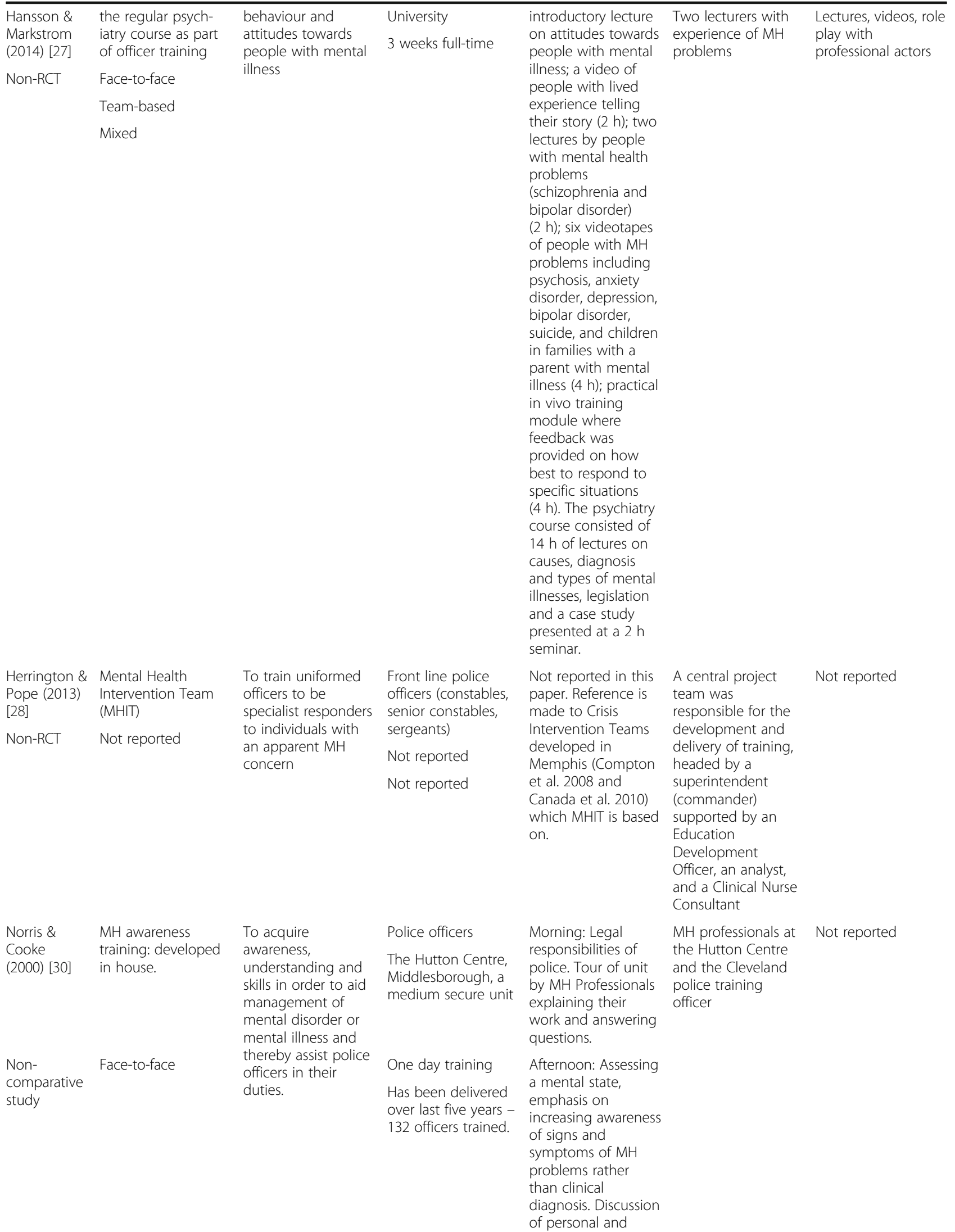


Table 3 Characteristics of training interventions (Continued)

\begin{tabular}{|c|c|c|c|c|c|c|}
\hline & & & & $\begin{array}{l}\text { professional } \\
\text { experiences and } \\
\text { interactive role plays } \\
\text { and teaching. }\end{array}$ & & \\
\hline & \multicolumn{3}{|l|}{ Team based } & \multirow[b]{2}{*}{$\begin{array}{l}\text { The training } \\
\text { emphasised the } \\
\text { "distinction between } \\
\text { a 'criminal' and a } \\
\text { 'mentally disordered } \\
\text { offender"', and the } \\
\text { "need for care and } \\
\text { treatment as } \\
\text { opposed to } \\
\text { incarceration and } \\
\text { punishment" }\end{array}$} & & \\
\hline & \multicolumn{3}{|l|}{ Mixed } & & & \\
\hline $\begin{array}{l}\text { Pinfold et al. } \\
\text { (2003) [31] }\end{array}$ & \multirow{3}{*}{$\begin{array}{l}\text { Reducing psychiatric } \\
\text { stigma and } \\
\text { discrimination: } \\
\text { developed in house }\end{array}$} & \multirow{6}{*}{$\begin{array}{l}\text { To communicate the } \\
\text { ordinariness of } \\
\text { mental ill-health and } \\
\text { to address fear and } \\
\text { misunderstanding } \\
\text { surrounding experi- } \\
\text { ences labelled 'se- } \\
\text { vere mental illness'. } \\
\text { Raising participant } \\
\text { awareness; increas- } \\
\text { ing level of know- } \\
\text { ledge; changing } \\
\text { views and affecting } \\
\text { behaviour. }\end{array}$} & Police officers & \multirow{3}{*}{$\begin{array}{l}\text { Workshop 1: 'What } \\
\text { are MH problems?' } \\
\text { Including hearing } \\
\text { voices simulation } \\
\text { exercise a session on } \\
\text { recovery and talks } \\
\text { by an individual } \\
\text { explaining what it } \\
\text { feels to be } \\
\text { psychotic. }\end{array}$} & \multirow{3}{*}{$\begin{array}{l}\text { Developed by } \\
\text { project team, police } \\
\text { force, Rethink severe } \\
\text { mental illness } \\
\text { trainers. }\end{array}$} & \multirow{6}{*}{$\begin{array}{l}\text { Workshops } \\
\text { supported by } \\
\text { information packs }\end{array}$} \\
\hline \multirow{5}{*}{$\begin{array}{l}\text { Non- } \\
\text { comparative } \\
\text { study }\end{array}$} & & & $\begin{array}{l}\text { Two police areas in } \\
\text { Kent }\end{array}$ & & & \\
\hline & & & $\begin{array}{l}2 \times 2 \text { h workshops } \\
\text { over } 6 \text { month } \\
\text { period. }\end{array}$ & & & \\
\hline & Face-to-face & & & Workshop 2: 'How & Delivered by: service & \\
\hline & Team based & & & $\begin{array}{l}\text { can the pollce } \\
\text { support people with }\end{array}$ & $\begin{array}{l}\text { users, carers, social } \\
\text { workers, voluntary }\end{array}$ & \\
\hline & Mixed & & & $\begin{array}{l}\text { MH problems?' } \\
\text { including case } \\
\text { studies and talks } \\
\text { from carers and } \\
\text { service users } \\
\text { highlighting best } \\
\text { practice principles. } \\
\text { Additionally, } \\
\text { workshop two } \\
\text { reviewed mental } \\
\text { health act } 1983 \\
\text { legislation, local } \\
\text { service provision } \\
\text { and officers own MH } \\
\text { needs }\end{array}$ & sector staff & \\
\hline $\begin{array}{l}\text { Rafacz (2012) } \\
{[17]}\end{array}$ & $\begin{array}{l}\text { An on-line anti- } \\
\text { stigma program de- }\end{array}$ & $\begin{array}{l}\text { The control } \\
\text { condition aimed to }\end{array}$ & $\begin{array}{l}\text { Campus police } \\
\text { officers }\end{array}$ & $\begin{array}{l}\text { A } 17 \text { min long video } \\
\text { in which the }\end{array}$ & $\begin{array}{l}\text { Videos presented by } \\
\text { men with similar }\end{array}$ & $\begin{array}{l}\text { Video presentation } \\
\text { delivered online }\end{array}$ \\
\hline $\mathrm{RCT}$ & $\begin{array}{l}\text { livered in two differ- } \\
\text { ent ways: personal }\end{array}$ & $\begin{array}{l}\text { Increase general } \\
\text { knowledge of }\end{array}$ & Online & $\begin{array}{l}\text { presenter disclosed } \\
\text { his mental illness }\end{array}$ & $\begin{array}{l}\text { attributes to each } \\
\text { other, however in }\end{array}$ & \\
\hline & $\begin{array}{l}\text { experience vs infor- } \\
\text { mation giving }\end{array}$ & $\begin{array}{l}\text { specific MH } \\
\text { conditions. The }\end{array}$ & $17 \min$ & $\begin{array}{l}\text { (schizo-affective } \\
\text { disorder), and shared }\end{array}$ & $\begin{array}{l}\text { the intervention } \\
\text { group the presenter }\end{array}$ & \\
\hline & Online/web-based & $\begin{array}{l}\text { Intervention } \\
\text { condition aimed to }\end{array}$ & & $\begin{array}{l}\text { his initial } \\
\text { experiences of the }\end{array}$ & $\begin{array}{l}\text { disclosed having a } \\
\text { diagnosis of schizo- }\end{array}$ & \\
\hline & Self-directed & change attitudes & & illness and treatment & affective disorder & \\
\hline & Didactic & increased empathy) & & $\begin{array}{l}\text { where he is now } \\
\text { and his successes, } \\
\text { hopes and dreams } \\
\text { for the future. He } \\
\text { also discussed the } \\
\text { interaction he had } \\
\text { with police officers } \\
\text { and their effect on } \\
\text { the outcomes of his } \\
\text { illness. }\end{array}$ & & \\
\hline Police officers & : Interventions with & cific mental health & Cus & & & \\
\hline $\begin{array}{l}\text { Bailey et al. } \\
\text { (2001) [26] }\end{array}$ & & $\begin{array}{l}\text { Training aimed to } \\
\text { raise the awareness }\end{array}$ & & $\begin{array}{l}\text { Involved a role- } \\
\text { played exercise }\end{array}$ & $\begin{array}{l}\text { One of the co- } \\
\text { authors, a }\end{array}$ & $\begin{array}{l}\text { Briefing, role play, } \\
\text { plenary group. Other }\end{array}$ \\
\hline
\end{tabular}


Table 3 Characteristics of training interventions (Continued)

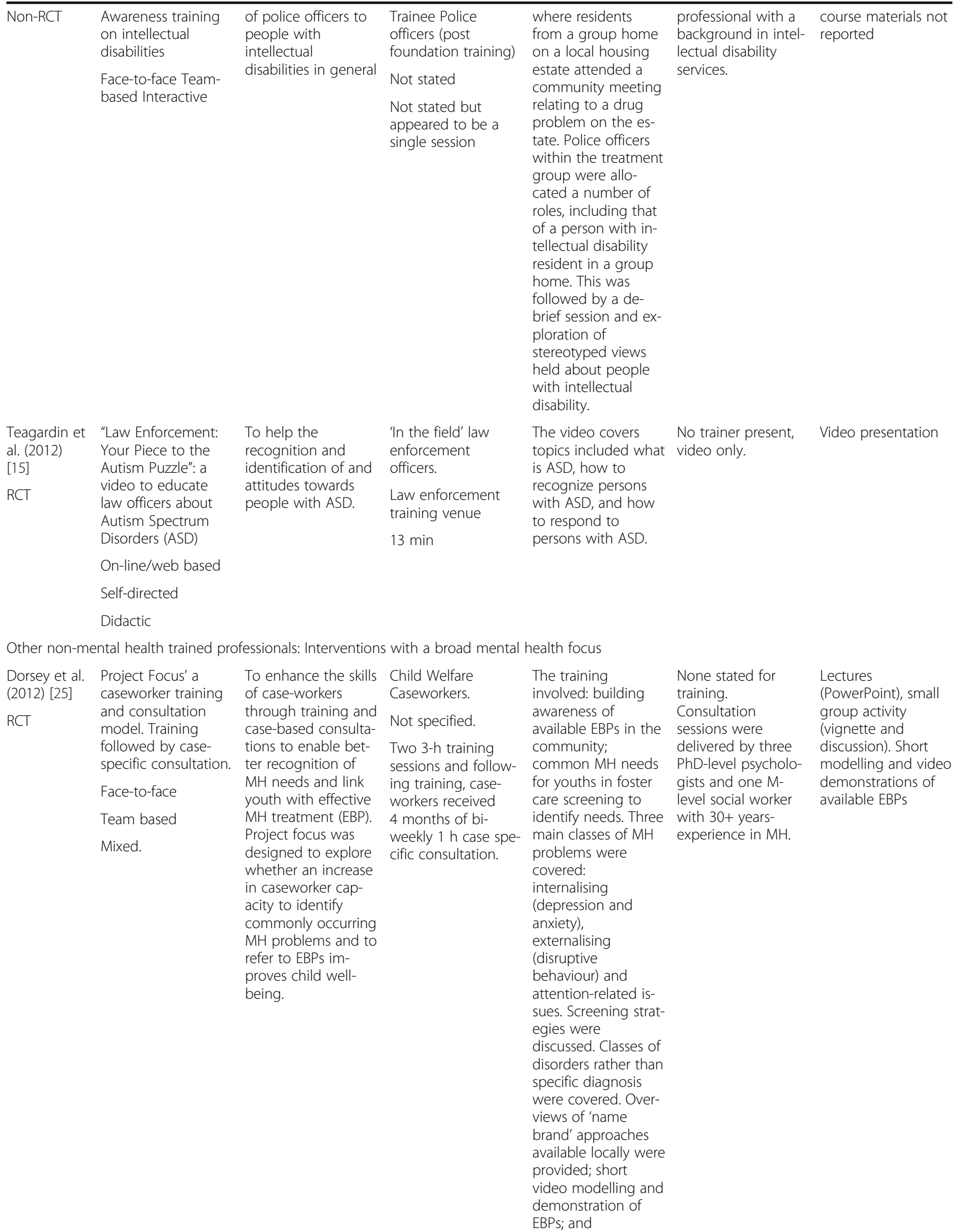


Table 3 Characteristics of training interventions (Continued)

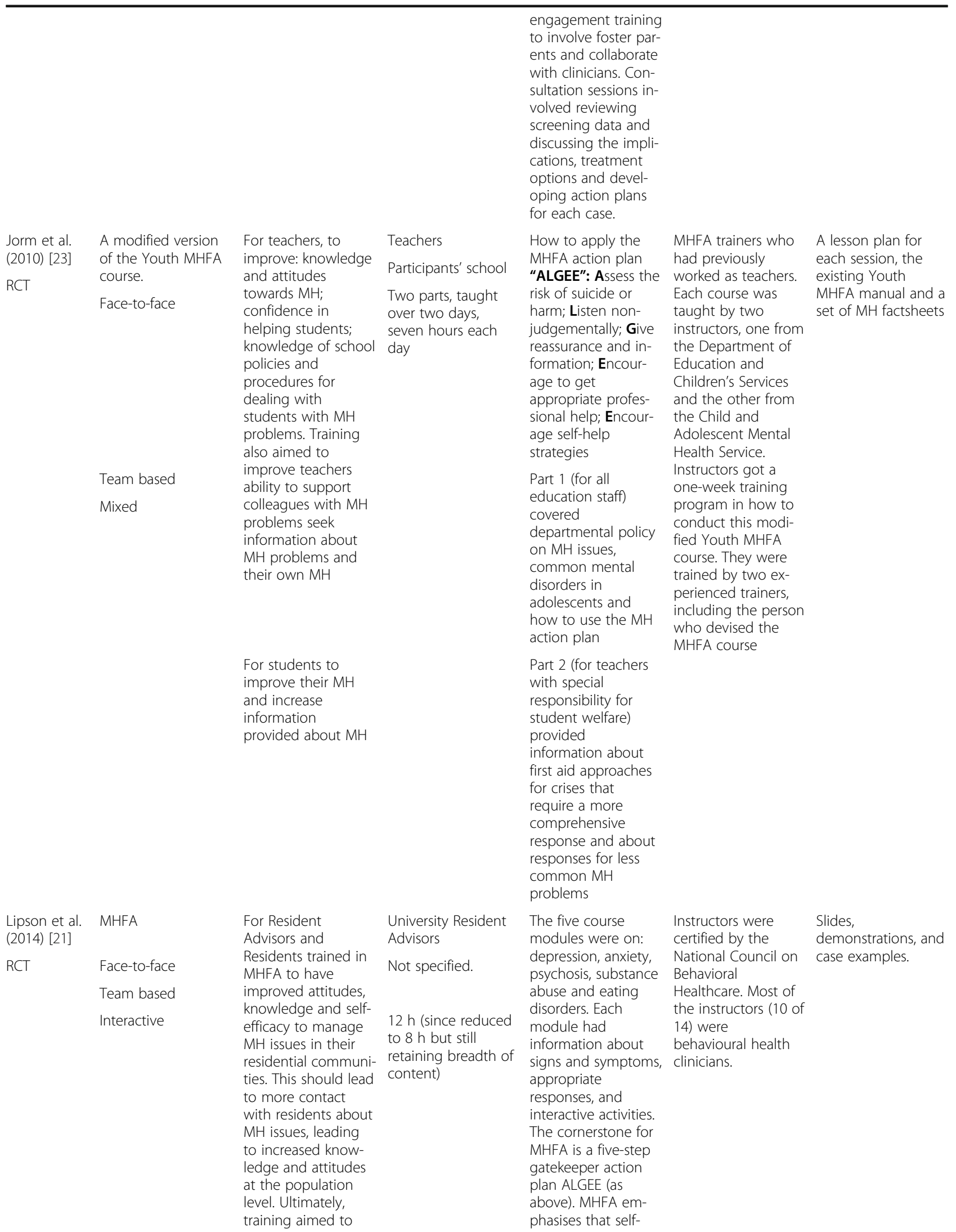


Table 3 Characteristics of training interventions (Continued)

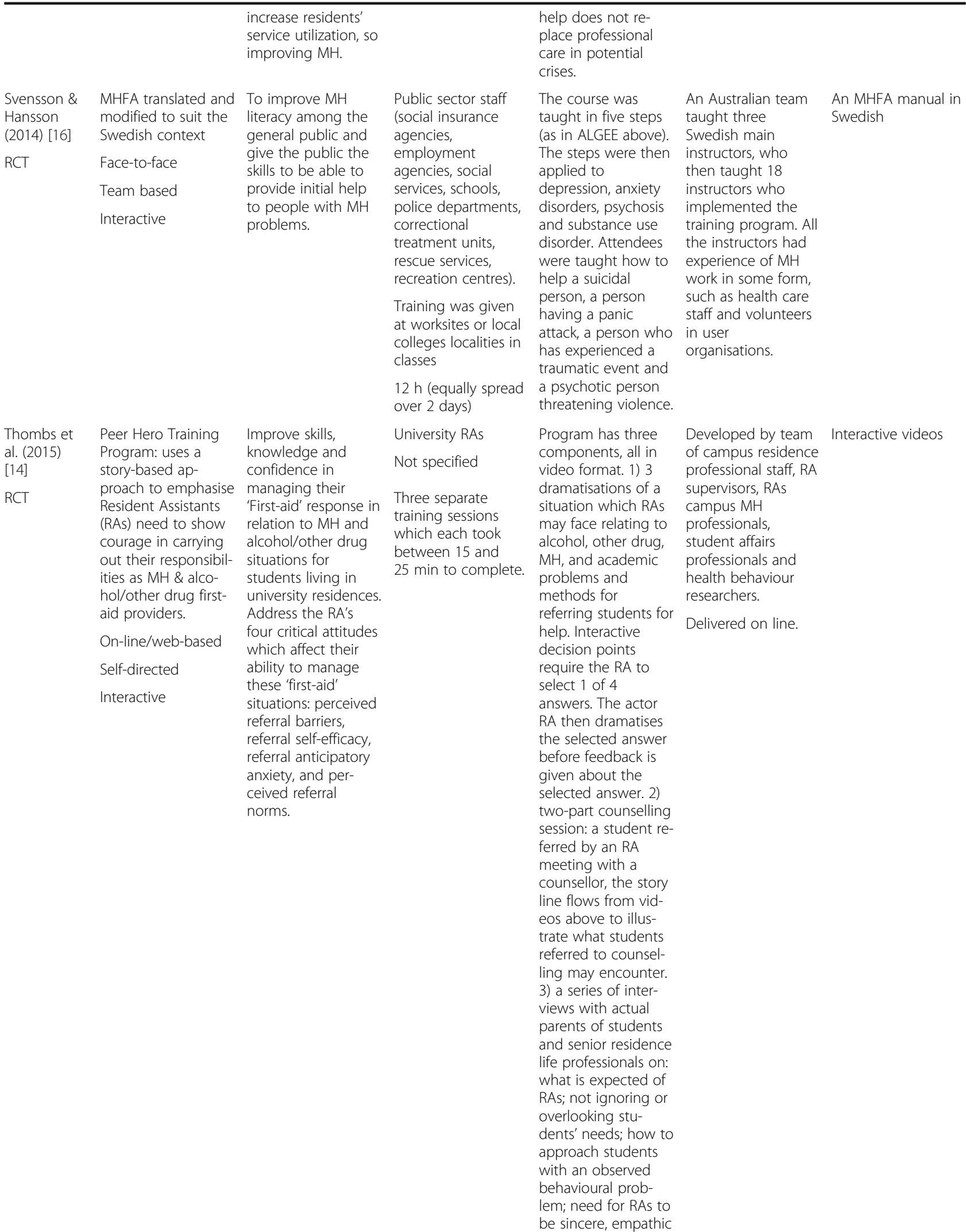


Table 3 Characteristics of training interventions (Continued)

and maintain

confidentiality.

Other non-mental health trained professionals: Interventions with a specific mental health focus

\begin{tabular}{|c|c|c|c|}
\hline Hart \& More & Information relating & Improve knowledge & Trainee teachers \\
\hline ] & $\begin{array}{l}\text { to Autism Spectrum } \\
\text { Disorder (ASD). }\end{array}$ & $\begin{array}{l}\text { Of ASD by } \\
\text { comparing two }\end{array}$ & Classroom \\
\hline RCT & On-line/web-based & $\begin{array}{l}\text { methods of } \\
\text { information delivery. }\end{array}$ & $20 \mathrm{~min}$ \\
\hline & Self-directed & & \\
\hline & Didactic & & \\
\hline
\end{tabular}

\begin{tabular}{|c|c|c|}
\hline $\begin{array}{l}\text { Kolko et al. } \\
\text { (2012) [22] }\end{array}$ & $\begin{array}{l}\text { Alternatives for } \\
\text { Families: A }\end{array}$ & $\begin{array}{l}\text { Provide practitioners } \\
\text { with skills to use AF- }\end{array}$ \\
\hline RCT & $\begin{array}{l}\text { Cognitive-Behavioral } \\
\text { Therapy (AF-CBT) }\end{array}$ & $\begin{array}{l}\text { CBT when working } \\
\text { with families with } \\
\text { physical forces, ag- } \\
\text { gression or abuse of } \\
\text { children. }\end{array}$ \\
\hline
\end{tabular}

Team based

Interactive
The ASD-related content was based on information on the Centers for Disease Control website and the course text, included: early warning signs and current prevalence and definition of ASD. Content used reflected the local cultural and linguistic diversity and that seen in ASD: this focused on potential underservice of ethnic and linguistic minority populations and teacher strategies for developing cultural competence and collaborative relationships with families.

Initial training based on the AF-CBT Session Guide: included didactic and experiential activities, case examples, group discussion, videotape

reviews, and behavioural rehearsal/

challenge exercises.

The session guide provides clinicians with an outline and examples for presenting the three phases of AF-CBT: engagement and psycho-education, individual skillbuilding, and family applications. Followup training: Each consultation began with a review of one or more AF-CBT topics, followed by two case presentations, feedback from consultants and the group, and problem solving to address the needs of the presenting clinicians. Booster training: Sessions focused on case conceptualisation, review of a skill topic, exploration of treatment adaptations and use of
The content of the For both groups training was material was located developed by the on a university authors: no details Blackboard Course provided. Delivery Management was online. Learning System which students were asked to log onto via a laptop. Those allocated to the podcast also used headphones.

Three experienced, Treatment book; AF'second generation' CBT session guide; trainers either alone clinician-friendly or in tandem (1 handouts; two chiltrainer to 8-15 dren's books practitioners).

Experienced AF-CBT clinicians, trainers, and developers generated the initial training content based on the AFCBT Session Guide. 
Table 3 Characteristics of training interventions (Continued)

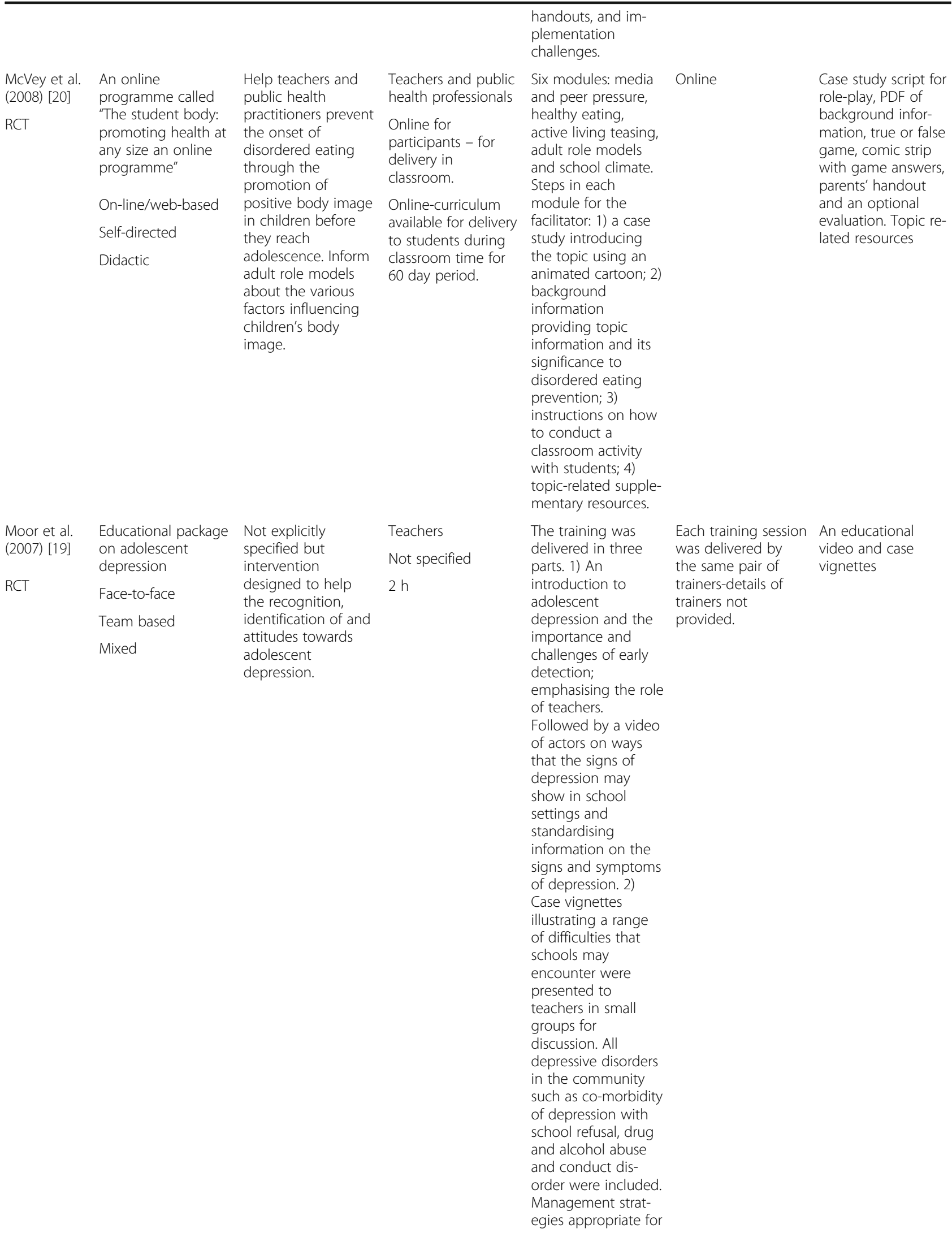




\begin{tabular}{|c|c|c|c|c|c|c|}
\hline & & & & $\begin{array}{l}\text { teachers to use in } \\
\text { school were cov- } \\
\text { ered, including prob- } \\
\text { lem solving } \\
\text { approaches. 3). Dis- } \\
\text { cussion of issues } \\
\text { specific to each staff } \\
\text { group's local triage } \\
\text { procedures and re- } \\
\text { ferral of hypothetical } \\
\text { at-risk pupils. }\end{array}$ & & \\
\hline \multirow{5}{*}{$\begin{array}{l}\text { Ostberg \& } \\
\text { Rydell (2012) } \\
\text { [18] } \\
\text { RCT }\end{array}$} & \multirow{2}{*}{$\begin{array}{l}\text { A modified version } \\
\text { of Barkley's parent } \\
\text { training programme- } \\
\text { adapted to setting } \\
\text { and for use with } \\
\text { teachers }\end{array}$} & \multirow{4}{*}{$\begin{array}{l}\text { To better equip } \\
\text { teachers and parents } \\
\text { with the 'tools' they } \\
\text { need and "strategies } \\
\text { in Everyday life" to } \\
\text { help children with } \\
\text { ADHD }\end{array}$} & $\begin{array}{l}\text { Teachers and } \\
\text { parents }\end{array}$ & \multirow{5}{*}{$\begin{array}{l}\text { Sessions from } \\
\text { Barkley's parent } \\
\text { training included } \\
\text { information about } \\
\text { neuropsychiatric } \\
\text { problems, teaching } \\
\text { participants to use } \\
\text { reinforcements, } \\
\text { problem solving and } \\
\text { communication with } \\
\text { the children. } \\
\text { Adaptations to the } \\
\text { programme } \\
\text { included: removal of } \\
\text { 'time-out' for } \\
\text { unwanted } \\
\text { behaviour, home } \\
\text { assignments were } \\
\text { based on the } \\
\text { problems parents } \\
\text { and teachers } \\
\text { experienced and } \\
\text { reported on. } \\
\text { Problem-solving as- } \\
\text { pect of the training } \\
\text { was extended. A } \\
\text { structure for co- } \\
\text { operation between } \\
\text { home and school } \\
\text { was formed. }\end{array}$} & \multirow{5}{*}{$\begin{array}{l}\text { Two "well-trained" } \\
\text { group-leaders per } \\
\text { group }\end{array}$} & \multirow{5}{*}{$\begin{array}{l}\text { Home assignments } \\
\text { based on problems } \\
\text { reported by parents } \\
\text { and teachers }\end{array}$} \\
\hline & & & $\begin{array}{l}\text { Child and } \\
\text { adolescent } \\
\text { psychiatry clinic. }\end{array}$ & & & \\
\hline & Face-to-face & & $\begin{array}{l}\text { Parents } 10 \text { weekly } \\
2 \text { h sessions, }\end{array}$ & & & \\
\hline & Team based & & teachers 8 sessions. & & & \\
\hline & Mixed & & & & & \\
\hline
\end{tabular}

made no difference to their practice. Although $77 \%$ of officers perceived an increase in their knowledge, there were no significant changes in general knowledge of mental illness and schizophrenia. Positive changes in attitude towards people with mental health problems were seen; however, the sessions did not impact on the officers' view that people with mental health problems are likely to be violent (61\% agreed at baseline, $54 \%$ at follow-up).

\section{Training for police officers: Interventions with a specific mental health focus}

One RCT [15, 18-20, 22, 24] and one non-RCT [26] focussed on training police officers to deal with people with specific mental health conditions.

Bailey et al. (2001) undertook a non-RCT of intellectual disabilities awareness training for probationary police officers [26]. The training was team-based, interactive and delivered face-to-face at the police training centre. The control group received no specific information about people with intellectual disabilities. The pre and post training evaluation showed a statistically significant improvement in attitudes in the intervention group compared with the control group.

One RCT investigated online delivered self-directed, didactic training for police officers about autism. Teagardin et al. (2012) compared front line police officers viewing a video about autism with a control group who did not see the video [15]. Significant differences before and after training were identified for reported confidence in identifying persons with Autism Spectrum Disorder (ASD) and in interacting with persons with ASD. Intervention group knowledge of ASD also significantly improved compared with control group post training.

Training for other non-mental health trained professionals: Interventions with a broad mental health focus

Dorsey et al. 's (2012) RCT examined whether a child welfare caseworker training and consultation model 
would improve knowledge of evidence based practice and ability to identify mental health problems and referral options [25]. Delivered face-to-face, the training was team based and included didactic and interactive elements. This was followed by 4 months of bi-weekly case specific support from a psychologist or social worker. Although the intervention group had significantly increased awareness of evidence based practice, the authors found no significant changes in practice or skills between the intervention and control groups.

The Mental Health First Aid (MHFA) programme started in Australia and has spread to other countries. Aimed originally at training adults in the general population to assist other adults, MHFA has been tailored for use by specific groups. We identified three RCTs evaluating MHFA for teachers [23], university resident advisors [21], and public sector staff such as social workers, human resource managers and employment managers [16]. All three study interventions were team based, involved interactive elements, and were delivered face-toface, over similar timeframes (12 to 14 h over 2 days). There were no changes in practice detected for MHFA trained resident advisors, nor in take up of mental health services by the students in the care of the resident advisors [21]. However, public sector staff who received the training improved their readiness to provide help to people in mental health crisis compared with the control group, which was sustained at 2 year follow-up [16]. Additionally students of trained teachers were significantly more likely to report that they received information about mental health problems than students of un-trained teachers. Improved attitudes in the intervention group were identified in two studies [16, 23], but no effect in the third [21]. All three studies reported increases in self assessed confidence and knowledge compared with their control groups.

One cluster RCT assessed the Peer Hero Training program, a story-based approach, which was delivered in interactive video format, with self-directed learning to university resident advisors [14]. At 7 month follow-up, the resident advisors reported making more than ten times as many first-aid encounters in the past 30-days for alcohol, drug, mental health, and academic problems compared with resident advisors assigned to training-as-usual. They also reported increased confidence and skills.

Training for other non-mental health trained professionals: Interventions with a specific mental health focus

One RCT investigated online self-directed, didactic training about autism. Hart and More's (2013) study provided information to student teachers; the group viewing a Podcast performed statistically significantly better on the ASD comprehension test compared with the comparator group who received the same information in written format [24].
This RCT was the only included study to report an underpinning theory that was successfully applied throughout the study [24].

A face-to-face, team based, interactive intervention, "Alternative for Families: A Cognitive-Behavioral Therapy (AF-CBT)" aimed to provide practitioners with skills to use AF-CBT when working with families where there is concern about physical discipline, aggression or abuse of children. The intervention was delivered to community practitioners and outcomes compared with a control group in an RCT by Kolko et al. (2012) [22]. Intervention participants reported high levels of satisfaction with all aspects of the training, in particular the training materials. Compared with the control group at 6 month follow-up, the AF-CBT group reported a significantly greater increase for the teaching processes; knowledge about CBT; skills in dealing with a history of abuse, and general psychological skills. However, at 18 months post training, these differences were no longer significant.

McVey et al. (2008) looked at the feasibility and usefulness of an on-line modular programme to help elementary school teachers and public health practitioners prevent eating disorders [20]. The didactic module was made available to the intervention group for self-directed study any time over a 60 day period: overall a high level of satisfaction with the modules was reported by participants. Compared with the comparator group, teachers in the intervention group reported statistically significant improvements over time in their knowledge about facts concerning restrictive dieting and about peer influences. There were no significant differences between public health professionals for knowledge items; however the intervention group did demonstrate significant increases in self-efficacy to fight weight bias compared with the comparator group. Almost all (94\%) of the intervention participants said the information learned would prompt them to make changes to their school environment; $74 \%$ said the program had positively influenced their own feelings about their body shape; and $93 \%$ reported that the program improved their overall delivery of body image and health eating curriculum to students.

Teachers were randomised to receive an educational package on adolescent depression or waiting list in an RCT by Moor et al. (2007) [19]. The 2 hour training was delivered face-to-face, was team based and included mixed presentation methods. Teacher responses to an attitude questionnaire showed increased confidence compared with the control group, but this did not translate into improved recognition of depressed pupils. Teachers in the experimental group recognized $52 \%$ of cases before the intervention and $45 \%$ afterwards, whilst the control teachers recognized $41 \%$ and $43 \%$, respectively. The training produced no improvement in recognition of depressed pupils. 
An RCT examined an existing parent training programme on Attention Deficit Hyperactivity Disorder (ADHD) adapted for delivery in a clinic and for use with teachers and parents [18]. Delivered face-to-face, the team based training included interactive and didactic elements. At 3 month follow up a significant reduction in parent-rated ADHD symptoms and in problematic behaviours were reported in the intervention group. For teacher ratings, significantly reduced emotional problems were found in the control group.

\section{Audits and evaluations carried out by police forces in England and Wales}

We received 25 responses from 22 different counties after sending out 75 email requests for audits of police training in mental health. However, this did not provide further data and no audits or evaluations have to our knowledge been undertaken.

\section{On-going or completed but not yet published work}

Our searches found a protocol for a cluster RCT on an integrated workplace mental health intervention [32]. The study is investigating whether improved leadership skills and mental health literacy leads to improved psychosocial working conditions for police officers in Southeast Australia. The ISRCTN registration lists the overall trial end date as 30/12/2016.

\section{Discussion}

We identified evaluations of a wide variety of training interventions, populations and settings. The training programmes ranged between awareness raising, ways to change practice, and comparison of training delivery methods. The interventions also varied from addressing specific mental health conditions to providing a broad understanding of mental health illnesses and vulnerabilities, with some including how to interact effectively. There were huge variations in the design, delivery method and content of the training, and in the knowledge, experience and skills of those developing and/or delivering the training. Although 12 RCTs were identified, overall the quality of reporting makes it difficult to assess the reliability of their findings.

A number of the training interventions included dramatisations or role play, some with actors or service users. These studies found some positive effect compared with their comparison group [14, 19, 21, 22, 26, 27]. Likewise, the non-comparative studies all included role play and all reported improvement in at least one outcome [29-31]. There are a large number of reviews on adult learning practices and methods [33-36]. Dunst and Trivette (2012) reviewed 58 RCTs and found that training using a variety of presentation methods in groups of less than 40, in applied settings, over $20 \mathrm{~h}$ on multiple occasions was optimum for acquiring new knowledge or skills [36]. Where details were reported, most of the interventions included in this review were delivered in line with evidence based best practice.

The trainers who deliver a training package are crucial to the success of changing perceptions and behaviours. Coleman and Cotton (2014), highlight the important role of the trainer in not only delivering effective training but in the success of implementing any related programme [37].The studies of training police officers nearly all used police trainers alongside mental health professionals in delivery of interventions: potentially helping each profession to understand the others organisational culture.

A dilemma when providing training on mental health issues to non-mental health professionals is deciding what trainees need to know and in what detail. There were no studies directly comparing general versus specific training programmes. The gaps in reporting details and the wide variation in the included studies precludes drawing even tentative conclusions about general mental health awareness raising versus condition specific training programs. Over half the studies had specific mental health foci, settings and participants limiting the generalisability of the findings. For example, learning disabilities frequently co-occur with mental ill health, but many of the training interventions focussed on one specific mental health issue without consideration of the potential for other vulnerabilities. Conversely, some of the included studies used pre-existing training packages, but with little reference to previous evaluations. For example, CIT is being rolled out across the USA and elsewhere but there is little robust evidence of its effectiveness [38, 39]. Likewise, the TEMPO (Training and Education about Mental illness for Police Organizations) model for Canadian police personnel, while developed on sound principles and research evidence, has not yet had the rigorous outcome evaluation of implementation recommended by Coleman and Cotton (2014) [37]. None of the studies identified aimed to provide skills that could be used in multiple situations.

\section{Limitations of this review}

While our searches were comprehensive it is possible that we failed to find some relevant studies. There were variations in the usefulness of tagging/thesaurus terms used in the databases searched. For example a paper in Criminal Justice Abstracts with the phrase "the impact of police training in mental health" in the title, used the thesaurus terms, "MENTAL health services/MENTAL illness/PEACE officers/POLICE reform", with no mention of training at all. This risked the paper being missed in the combination of "police AND training"; however the paper was identified in our hand searching. The search strategies were tailored to each individual database, but there is always a balance between sensitivity 
and specificity. Our use of web searches, forward and backward reference searches and the request for published or unpublished audits and/or evaluations from police trainers in England and Wales may have gone someway to mitigating the risk of publication bias.

Generalisability is limited as the searches were restricted to English language and studies in OECD countries. The participants, settings and interventions were in some cases very specialist, also limiting generalisability $[16,18,20,24,25]$.

Our research question was broad, therefore the evaluations we identified included a wide variety of training interventions, populations and settings; this could be considered a limitation. We found an overall lack of high quality evidence to inform training decisions for any non-mental health professionals coming into contact with people with a mental health issue.

Given the lack of RCTs of training specifically for police officers, we included methodologically less robust, nonrandomised controlled trials: and restricted their inclusion to those in the police setting. Even within the RCTs, the heterogeneity in all aspects of the included studies made a narrative synthesis the only option, limiting the strength of the conclusions that can be drawn. While the outcome categories selected are widely used in research of training, they presented some interpretation challenges when data extracting. This was mainly because of the diversity of outcomes assessed in the included studies and a general lack of detail in the reporting of the results.

Reporting was generally incomplete, but we did not have the resources to contact authors in the hope of receiving further details.

\section{Implications for future research}

There is a need for high quality RCTs to evaluate the impact of training programmes for non-mental health trained professionals coming into contact with people with mental health issues, and in police officers in particular. A 2012 study in a police district of 198,000 inhabitants in the Netherlands linked police data with mental health care information [40]. In 1 year the police dealt with 492 crisis situations, and in half those cases the individuals were disengaged from mental health services. The findings confirmed the important role of police officers in linking people with mental ill health to care, and the necessity for appropriate training and understanding of local mental health services and resources for front line police.

We were interested in seven well established expected outcomes of training; however many were not measured in the evaluations identified. The few studies that attempted to measure a change in outcomes for the groups of people the trainees came into contact with found little or no impact $[21,23,28]$. Obtaining good quality service user insight into the effectiveness of training is challenging, particularly when the interaction is with the police. A surrogate measure could be community satisfaction performance indicators related to mental health interactions, as recommended by The Lawrence inquiry (1999) [41]. The short follow up time was an acknowledged limitation in most of the studies. A variety of evaluation tools were used, mostly designed inhouse or where a validated tool was used this was modified in some way. A recent systematic review of the measurement properties of tools measuring mental health knowledge recommends using tools with an evidence base which reach the threshold for positive ratings according to the COSMIN checklist [42].

Outcomes measured should include those important for the trainees' roles and for the people with mental health issues. The development of a set of core outcome measures as part of and/or to inform future studies would be beneficial. The COMET initiative has a database of core outcome measures in effectiveness trials [43]. For example The Engager 2 project is developing a set of outcome measures as part of a larger project to develop and evaluate a complex intervention for prisoners with common mental health problems who are coming to the end of their sentence [44].

Future research would also benefit from more complete reporting. The Equator Network provides a single point of access to a range of useful documents [45] including the TIDierR checklist [46] and CONSORT statement [47] for the accurate reporting of interventions and RCTs respectively. Checklists and guidelines for reporting protocols and other types of studies are also available. Training interventions can include multiple elements, and be influenced by the characteristics of the setting and context in which it is being delivered and implemented. As such they may be considered complex interventions, for which specific reporting guidelines are also available [48].

\section{Conclusions}

A variety of training programmes exist for non-mental health professionals who come into contact with people who have mental health issues. There may be some short term change in behaviour for the trainees, but longer term follow up is needed. Research evaluating training for UK police officers is needed in which a number of methodological issues need to be addressed.

\section{Additional files}

Additional file 1: Search strategies. (DOCX $74 \mathrm{~kb}$ )

Additional file 2: Table of studies excluded on review of full paper. (DOCX $50 \mathrm{~kb}$ )

Additional file 3: Quality assessments. (DOCX 19 kb)

\section{Abbreviations}

ACPO: Association of Chief Police Officers; ADHD: Attention deficit hyperactivity disorder; AF-CBT: Alternatives for Families: A CognitiveBehavioural Therapy; ASD: Autism Spectrum Disorder; CIT: Crisis Intervention 
Team; COMET: Core Outcome Measures in Effectiveness Trials; CONSORT: Consolidated Standards of Reporting Trials; DH: Department of Health; ES: Effect size; ISRCTN: International Standard Registered Clinical/ soCial sTudy Number; MHFA: Mental Health First Aid; MHIT: Mental Health Intervention Team; NPIA: National Policing Improvement Agency; OECD: Organisation for Economic Co-operation and Development; OR: Odds ratio; PROSPERO: International Prospective Register of Systematic Reviews; RCT: Randomised Controlled Trial; ROBIS: Risk of bias in systematic reviews; TIDierR: Template for Intervention Description and Replication; USA: United States of America

\section{Acknowledgements}

The following collaborators are thanked for their comments on the draft protocol: Richard Anderson, North Yorkshire Police; David Torgerson, and Catherine Hewitt, York Trials Unit, University of York; Martin Webber, and Nicola Moran, Department of Social Policy and Social Work, University of York. The authors would like to thank Kat Chatterton for assistance with ILLs and formatting. The authors also extend their thanks and appreciation for the time taken by the police personnel who responded to the request for audit or evaluation information.

\section{Funding}

This review was undertaken as part of Connect, the Co-production of policing evidence, research and training: focus mental health project (http:// connectebp.org/). The project is funded by the Higher Education Funding Council for England (HEFCE) and the Home Office through the College of Policing.

\section{Availability of data and materials}

The data extraction and quality assessment tables are available on request.

\section{Authors' contributions}

$A B$ lead the review and was responsible for writing the protocol, study selection, data extraction, validity assessment and writing the final report. KW devised the search strategies and carried out the literature searches and wrote the search methodology section of the paper. $A B, A C$ and $A H-M$ undertook study selection, data extraction and validity assessment. AH-M undertook the forward and backward hand searches and searches of websites. All the authors commented on the draft protocol and the final report. CM provided input at all stages, commented on all draft documents and had overall responsibility for the review. All authors read and approved the final manuscript.

\section{Competing interests}

The authors declare that they have no competing interests.

\section{Consent for publication}

Not applicable.

\section{Ethics approval and consent to participate}

Not applicable to this systematic review.

\section{Publisher's Note}

Springer Nature remains neutral with regard to jurisdictional claims in published maps and institutional affiliations.

\section{Author details}

${ }^{1}$ York Trials Unit, Department of Health Sciences, Faculty of Science, University of York, Helslington, York YO10 5DD, UK. ${ }^{2}$ Centre for Reviews and Dissemination, University of York, Helslington, York, UK. ${ }^{3}$ North Yorkshire Police, Newby Wiske, UK

Received: 7 October 2016 Accepted: 15 May 2017

Published online: 25 May 2017

\section{References}

1. Her Majesty's Inspectorate of Constabulary, C.Q.C., Her Majesty's Inspectorate of Prisons, and Healthcare Inspectorate Wales. A criminal use of police cells? The use of police custody as a place of safety for people with mental health needs. June 2013, Her Majesty's Inspectorate of Constabulary, Care Quality Commission. London: Her Majesty's Inspectorate of Prisons, and Healthcare Inspectorate Wales.
2. Department of Health and Concordat signatories, Mental health crisis care concordat improving outcomes for people experiencing mental health crisis. February 2014

3. Mental Health Act 1983 Section 136. http://www.legislation.gov.uk/ukpga/ 1983/20/section/136 The Stationery Office. UK.

4. National Policing Improvement Agency on behalf of the Association of Chief Police Officers, Guidance on responding to people with mental ill health or learning disabilities. 2010.

5. Bluestone J, et al. Effective in-service training design and delivery: evidence from an integrative literature review. Hum Resour Health. 2013;11:51.

6. Hutchinson L. Evaluating and researching the effectiveness of educational interventions. BMJ. 1999;318(7193):1267-9

7. Khan KS, Coomarasamy A. A hierarchy of effective teaching and learning to acquire competence in evidenced-based medicine. BMC Medical Education. 2006;6:59.

8. Wilkes M, Bligh J. Evaluating educational interventions. BMJ: British Medical Journal. 1999;318(7193):1269-72.

9. Whiting $P$, et al. ROBIS: a new tool to assess risk of bias in systematic reviews was developed. J Clin Epidemiol. 2015;69:225-34.

10. Higgins JPT, et al. The Cochrane Collaboration's tool for assessing risk of bias in randomised trials. BMJ. 2011;343:d5928.

11. Wells GA, et al. The Newcastle-Ottawa Scale (NOS) for assessing the quality of nonrandomised studies in meta-analyses. 28 Apr 2016]; Available from: http:// www.ohri.ca/programs/clinical_epidemiology/oxford.htm.

12. National Institutes of Health: and National Heart Lung and Blood Institute. Quality assessment tool for before-after (pre-post) studies with no control group. Systematic Evidence Reviews and Clinical Practice Guidelines. Washington DC: National Institutes of Health; 2014.

13. Compton MT, et al. A comprehensive review of extant research on crisis intervention team (CIT) programs. J Am Acad Psychiatry Law. 2008;36(1):47-55.

14. Thombs D, et al. Resident assistant training program for increasing alcohol, other drug, and mental health first-aid efforts. Prev Sci. 2015;16(4):508-17.

15. Teagardin J, et al. Randomized trial of law enforcement training on autism spectrum disorders. Research in Autism Spectrum Disorders. 2012;6(3):1113-8.

16. Svensson B, Hansson L. Effectiveness of mental health first aid training in Sweden. A randomized controlled trial with a six-month and two-year follow-up. PLoS One. 2014;9(6):e100911.

17. Rafacz, J.D., Anti-stigma programs: stigma in campus police officers. Ann Arbor: ProQuest LLC; 2012

18. Ostberg M, Rydell AM. An efficacy study of a combined parent and teacher management training programme for children with ADHD. Nord J Psychiatry. 2012;66(2):123-30

19. Moor $\mathrm{S}$, et al. Improving the recognition of depression in adolescence: can we teach the teachers? J Adolesc. 2007;30(1):81-95.

20. McVey G, et al. A controlled evaluation of web-based training for teachers and public health practitioners on the prevention of eating disorders. Brunner-Mazel Eating Disorders Monograph Series. 2008; 17(1):1-26.

21. Lipson SK, et al. Gatekeeper training and access to mental health care at universities and colleges. J Adolesc Health. 2014;55(5):612-9.

22. Kolko DJ, et al. Implementation of AF-CBT by community practitioners serving child welfare and mental health: a randomized trial. Child Maltreatment. 2012;17(1):32-46.

23. Jorm AF, et al. Mental health first aid training for high school teachers: a cluster randomized trial. BMC Psychiatry. 2010;10:51.

24. Hart JE, More CM. Investigating the impact of technology on pre-service teacher knowledge of autism spectrum disorder. Education and Training in Autism and Developmental Disabilities. 2013:48(4):504-13.

25. Dorsey $\mathrm{S}$, et al. Child welfare caseworkers as service brokers for youth in foster care: findings from project focus. Child Maltreatment. 2012;17(1):22-31.

26. Bailey A, Barr O, Bunting B. Police attitudes toward people with intellectual disability: an evaluation of awareness training. J Intellect Disabil Res. 2001; 45(Pt 4):344-50.

27. Hansson L, Markstrom U. The effectiveness of an anti-stigma intervention in a basic police officer training programme: a controlled study. BMC Psychiatry. 2014;14:55

28. Herrington $\mathrm{V}$, Pope $\mathrm{R}$. The impact of police training in mental health: an example from Australia. Polic Soc. 2014;24(5):501-22.

29. Forni C, Caswell N, Spicer J. Delivering mental health awareness training to police officers. Nurs Times. 2009;105(10):12-3.

30. Norris C, Cooke E. Mental health training scheme for police officers. Professional Nurse. 2000;15(10):655-8. 
31. Pinfold V, et al. Reducing psychiatric stigma and discrimination-evaluating an educational intervention with the police force in England. Soc Psychiatry Psychiatr Epidemiol. 2003;38(6):337-44.

32. LaMontagne $A D$, et al. An integrated workplace mental health intervention in a policing context: protocol for a cluster randomised control trial. BMC Psychiatry. 2016;16:49.

33. Tusting K, Barton D. Models of adult learning: a literature review. Leicester: NIACE; 2006.

34. Taylor EW. An update of transformative learning theory: a critical review of the empirical research (1999-2005). Int J Lifelong Educ. 2007;26(2):173-91.

35. Marriott BR, et al. A methodology for enhancing implementation science proposals: comparison of face-to-face versus virtual workshops. Implement Sci. 2016;11(1):1-11.

36. Dunst CJ, Trivette CM. Moderators of the effectiveness of adult learning method practices. J Soc Sci. 2012;8(2):143-8.

37. Coleman T, Cotton D. TEMPO: a contemporary model for police education and training about mental illness. Int J Law Psychiatry. 2014;37(4):325-33.

38. Watson AC, Fulambarker AJ. The crisis intervention team model of police response to mental health crises: a primer for mental health practitioners. Best Practices in Mental Health. 2012;8(2):71.

39. Chappell D, O'Brien A. Police responses to persons with a mental illness: international perspectives. Int J Law Psychiatry. 2014;37(4):321-4.

40. van den Brink RH, et al. Role of the police in linking individuals experiencing mental health crises with mental health services. BMC Psychiatry. 2012;12(1):1-7.

41. Home Office. The Stephen Lawrence inquiry (Macpherson report). London: HMSO; 1999.

42. Wei $Y$, et al. Measurement properties of tools measuring mental health knowledge: a systematic review. BMC Psychiatry. 2016;16(1):1-16.

43. Williamson P, et al. Comet Initiative: Core Outcome Measures in Effectiveness Trials. 20 July 2016]; Available from: http://www.comet-initiative.org/.

44. Byng R, et al. Engager 2: Developing and evaluating a collaborative care intervention for offenders with common mental health problems, near to and after release. Comet Initiative: Core Outcome Measures in Effectiveness Trials 201320 July 2016]; Available from: http://www.comet-initiative.org/studies/ details/602.

45. The EQUATOR Network. Equator: Enhancing the QUAlity and Transparency Of health Research. 20 July 2016]; Available from: http://www.equator-network.org/.

46. Hoffmann TC, et al. Better reporting of interventions: template for intervention description and replication (TIDieR) checklist and guide. BMJ (Clinical research ed). 2014;348:91687.

47. Schulz KF, Altman DG, Moher D. CONSORT 2010 statement: updated guidelines for reporting parallel group randomised trials. PLoS Med. 2010; 7(3):e1000251.

48. Mohler R, Kopke S, Meyer G. Criteria for reporting the development and evaluation of complex interventions in healthcare: revised guideline (CReDECl 2). Trials. 2015;16:204.

\section{Submit your next manuscript to BioMed Central and we will help you at every step:}

- We accept pre-submission inquiries

- Our selector tool helps you to find the most relevant journal

- We provide round the clock customer support

- Convenient online submission

- Thorough peer review

- Inclusion in PubMed and all major indexing services

- Maximum visibility for your research

Submit your manuscript at www.biomedcentral.com/submit

) Biomed Central 